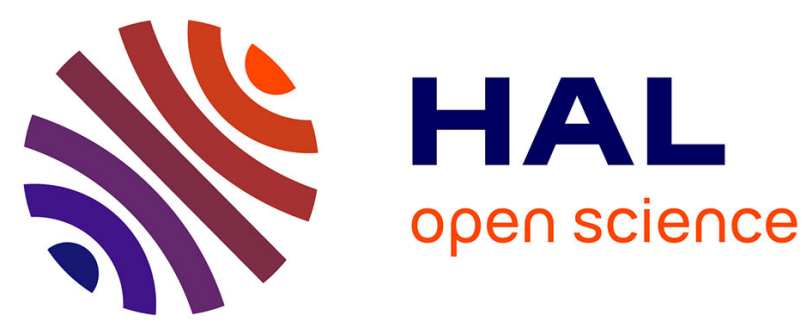

\title{
Le quartier de l'Arsenal à Metz (Moselle) : topographie urbaine et évolution architecturale durant l'Antiquité
} Dominique Heckenbenner, Philippe Brunella, Marc Leroy, Milan Milutinovic, Pierre Thion, Sabine Baccega, Jean-Marie Blaising, Isabelle Bourger, Olivier Faye, Murielle Georges-Leroy, et al.

\section{To cite this version:}

Dominique Heckenbenner, Philippe Brunella, Marc Leroy, Milan Milutinovic, Pierre Thion, et al.. Le quartier de l'Arsenal à Metz (Moselle): topographie urbaine et évolution architecturale durant l'Antiquité. Gallia - Fouilles et monuments archéologiques en France métropolitaine, 1992, 49, pp.935. 10.3406/galia.1992.2926 . hal-01913175

\section{HAL Id: hal-01913175 \\ https://hal.science/hal-01913175}

Submitted on 19 Jan 2020

HAL is a multi-disciplinary open access archive for the deposit and dissemination of scientific research documents, whether they are published or not. The documents may come from teaching and research institutions in France or abroad, or from public or private research centers.
L'archive ouverte pluridisciplinaire HAL, est destinée au dépôt et à la diffusion de documents scientifiques de niveau recherche, publiés ou non, émanant des établissements d'enseignement et de recherche français ou étrangers, des laboratoires publics ou privés.

\section{(ㅇ)(1) $\$$}

Distributed under a Creative Commons Attribution - NonCommercial - NoDerivatives| 4.0 


\title{
Le quartier de l'Arsenal à Metz (Moselle) : topographie urbaine et évolution architecturale durant l'Antiquité
}

\author{
par Dominique HECKENBENNER ${ }^{1}$, Philippe BRUNELLA ${ }^{2}$, Marc LEROY ${ }^{3}$, \\ Milan MILUTINOVIC ${ }^{4}$, Pierre THION ${ }^{5}$ \\ avec la collaboration de \\ Sabine BACCEGA ${ }^{6}$, Jean-Marie BLAISING ${ }^{7}$, Isabelle BOURGER $^{8}$, \\ Olivier FAYE (dessin) ${ }^{9}$, Murielle GEORGES-LEROY ${ }^{10}$, Ghyslaine GIRARD ${ }^{11}$, \\ Vincent KRIER ${ }^{12}$, Claude PEPE ${ }^{13}$, Claude VOIGNIER (relevé général) ${ }^{14}$
}

(1I Le quartier de l'Arsenal, situé au sud-ouest de la ville, a fait l'objet de deux fouilles de sauvetage récentes su: une surface cohérente de $5000 \mathrm{~m}^{2}$. En raison d'importants travaux de nivellement au xıx ${ }^{\mathrm{e}}$ s., les résultats ec) lus significatifs concernent les $\mathrm{I}^{\mathrm{er}}-\mathrm{III}^{\mathrm{e}} \mathrm{s}$.

Il a ainsi été possible, pour la première fois à Metz, d'appréhender le parcellaire et son évolution sur près du (iê: d'un îlot. A un habitat constitué de lots distincts d'environ $10 \mathrm{~m}$ de large, appuyés sur la rue, succède au II $_{\text {es. }}$. une propriété plus grande, entièrement occupée par une vaste domus.

L'élude à titre expérimental des traces organiques présentes dans les caniveaux de voirie s'avère prometteû̀se, et devrait notamment permettre de mieux comprendre la gestion des déchets en milieu urbain.

Enfin la mise au jour de quatre rues et d'un fossé d'une cinquième renouvelle considérablement nos connaissances sur l'organisation du quartier. A la lumière des données de fouilles anciennes se dessine maintenant un système original, distinct du réseau vraisemblablement orthonormé du centre-ville, et que le relief assez accidenté du site ne peut à lui seul expliquer.

1 Conservateur territorial du Patrimoine, Musée du Pays de Sarrebourg, 13, avenue de France, 57400 Sarrebourg.

2 Archéologue départemental adjoint, Hôtel du département, BP 1096, 57036 Metz Cedex.

3 Contractuel AFA.V, Service régional de l'archéologie de Lorraine, 6, place de Chambre, 57045 Metz Cedex 1.

4 Contractuel AFA.N, Service régional de l'archéologie de Lorraine, 6, place de Chambre, 57045 Metz Cedex 1.

5 Ingénieur d'études, Service régional de l'archéologie de Lorraine, 6, place de Chambre, 57045 Metz Cedex 1.

6 Contractuelle AFAN, Service régional de l'archéologie de Lorraine, 6, place de Chambre, 57045 Metz Cedex 1.

7 Contractuel AFA.N, Service régional de l'archéologie de Lorraine, 6, place de Chambre, 57045 Metz Cedex 1.

8 Contractuelle AFA.V, Service régional de l'archéologie de Lorraine, 6, place de Chambre, 57045 Metz Cedex 1.
9 Contractuel AFA.V, Service régional de l'archéologie de Lorraine, 6, place de Chambre, 57045 Metz Cedex 1.

10 Conservateur du Patrimoine, Service régional de l'archéologie de Lorraine, 6, place de Chambre, 57045 Metz Cedex 1.

11 Contractuelle AFA.V, Service régional de l'archéologie de Lorraine, 6, place de Chambre, 57045 Metz Cedex 1.

12 Contractuel AFA.V, Laboratoire de chrono-écologie de Besançon (UPR 7557), 16, route de Gray, 25030 Besançon Cedex.

13 Maitre de conférences à l'Université de Paris VI. Laboratoire de spectrochimie moléculaire, 4, place Jussieu, 75005 Paris.

14 Groupe universitaire messin de recherches archéologiques, 31, rue Saint-Maximin, 57070 Metz. 
The district of the Arsenal in the south-west of the town has recently been the subject of two extensive rescure excavations over a 5,000 sq. metres area. Due to a significant amount of levelling work in the 19th century, the most revealing results concern the years between the 1st and 3rd centuries.

It has, thus been possible for the first time in Metz to understand the layout of the parcels of land and its development over nearly a third of an insula. A settlement made up of distinct plots around 10 metres in size, facing the street, was followed by a larger estate in the 2nd century, totally occupied by a vast domus.

The preliminary sludy of organic traces found in the gutters of the streets looks promising and should, above all, lead to a belter understanding of the way rubbish was deall with in the urban area.

Finally the find of four streets and a ditch from a fifth one considerably adds to our knowledge of the organisation of the district. In conjunction with evidence from former excavations, it is now possible to trace an original system, seemingly different from the orthonormal network of the town centre.and which cannot just be explained by the rather hilly relief of the sile.

Mots clés : topographie urbaine, îlot, parcellaire, actus, habitat, architecture de terre et de bois, domus, portique, rue, caniveau, chimie organique, enduits peints, artisanat métallurgique, céramique, Metz, Moselle.

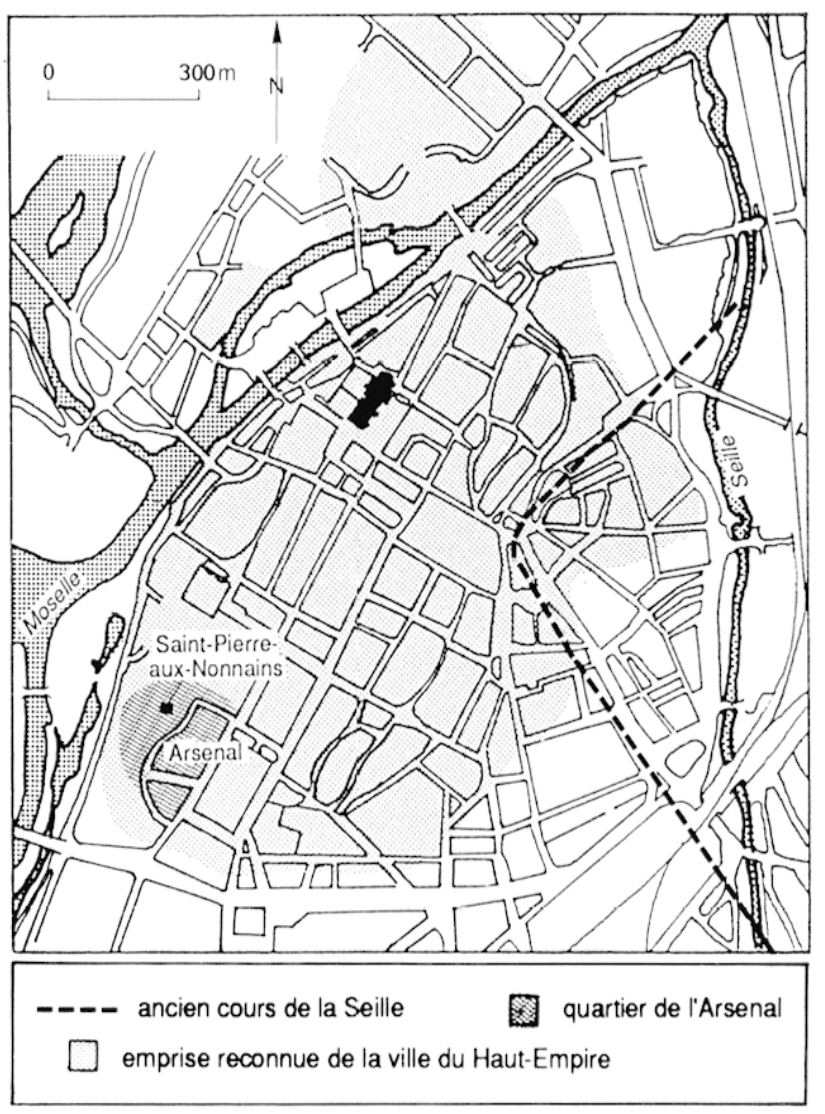

Fig. 1 - Situation du quartier de l'Arsenal.
L'antique Divodurum, chef-lieu de la cité des Médiomatriques, est située au confluent de la Moselle et de la Seille et au carrefour des voies Lyon-Trèves et Reims-vallée rhénane. Dès le milieu du $\mathrm{I}^{\mathrm{er}}$ s., la ville occupe non seulement le plateau d'interfluve, mais s'étend également au-delà du bras est de la Moselle et du cours ancien de la Seille (fig. 1). L'organisation nouvelle du limes sous les Flaviens et l'importance croissante de T'rèves, qui devient sans doute à la même époque la capitale provinciale, stimulent le développement de Metz, ville-étape et centre de ravitaillement pour les armées. Chef-lieu de cité prospère sur le plan commercial et économique, elle se dote de monuments publics (aqueduc, thermes, grand et petit amphithéâtres ...) (Frézouls, 1982).

L'antiquité tardive voit l'édification d'une enceinte urbaine, dont le tracé reconnu dans ses grandes lignes tire parti du relief tout en intégrant certains quartiers importants. La surface enclose, qui atteint 58 ha, témoigne d'une vitalité certaine de la cité au Bas-Empire.

Localisé au sud-ouest de la ville (fig. 1), le quartier de l'Arsenal a fait l'objet ces dernières années de plusieurs fouilles archéologiques de sauvetage, qui renouvellent profondément nos connaissances sur Metz antique.

L'intervention la plus importante a été motivée par le projet de la municipalité de transformer l'arsenal Ney en un vaste auditorium, aujourd'hui achevé, l'«Arsenal». 

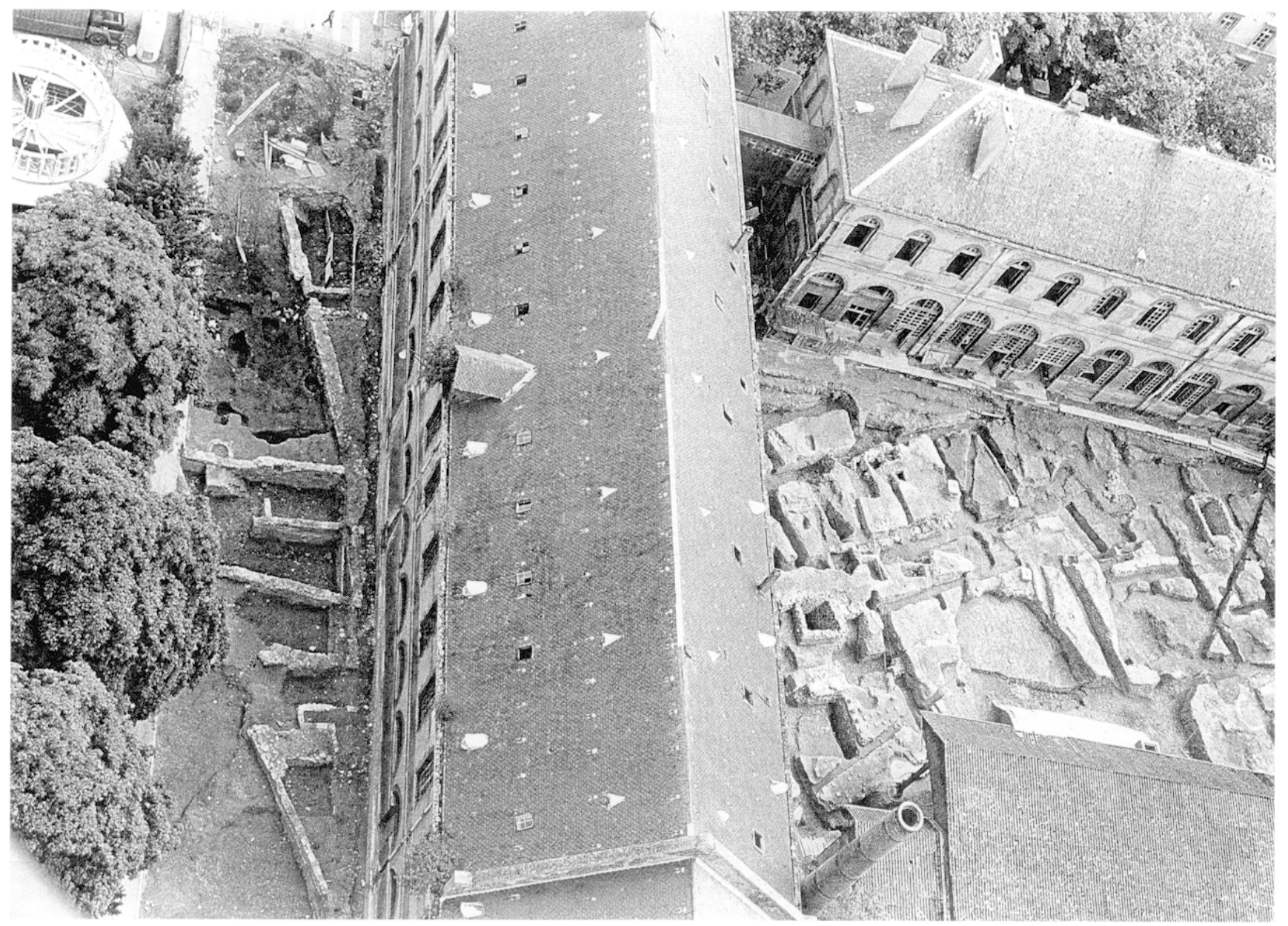

Fig. 2 - Vue générale des fouilles de l'Arsenal en 1986.

Ce programme a été à l'origine d'une première phase de sondages de reconnaissance (dirigés par Ph. Brunella en 1981), puis de trois campagnes de durée limitée (dirigées par H. Jannin en 1983 et par I). Heckenbenner en 1984 et 1985), suivies par une fouille de plus grande envergure, sur convention, menée d'avril à octobre 1986 par la Direction des antiquités de la circonscription de Lorraine, sous la responsabilité de I). Heckenbenner et de $\mathrm{P}$. Thion ${ }^{15}$.

15. Nous exprimons nos plus vifs remerciements à tous ceux. bénévoles du Groupe universitaire messin de recherches archéologiques et jeunes de l'Animation estivale, TIG, TLC, objecteurs de conscience et collegues qui ont pris part aux recherches. depuis le décapage jusqu'à la publication.

Toute notre gratitude va également à MII.J. Faudon, premier adjoint au maire de Metz et R. Tritschler, adjoint, délégué aux affaires culturelles.

Notre travail a été grandement facilité par MM. P. (iebler et P.-E. Wagner, alors respectivement conseiller tech-
Les terrassements prévus concernaient la totalité de la cour et deux des corps de bâtiment du XIX ${ }^{e}$ s., ainsi qu'une partie de la rue de la Citadelle, soit une surface totale de près de $6000 \mathrm{~m}^{2}$ (fig. 2 et 3 ).

Cette première opération fut suivie quelques mois plus tard par deux autres sauvetages effectués dans les environs de l'Arsenal et placés sous la direction de $\mathrm{Ph}$. Brunella. La première fouille, rendue nécessaire par la création d'un passage souterrain

nique et conseiller culturel à la Ville de Metz, dont l'amical soutien et l'intérèt pour nos recherches ont été constants.

L. appui des directeurs des antiquités de Lorraine successifs. MII. M. Colardelle. J.-L. Massy puis Mme J. Burnouf, a été décisif pour mener à bien cette operation. Nous tenons enfin à remercier tout particulièrement $M^{\mathrm{me}} \mathrm{Y}$. Brignon, qui a bien voulu assurer la saisie du manuscrit, ainsi que l’équipe du Rheinisches Landesmuseum de Trèves, qui a facilité notre travail à la bibliothèque du musée. 


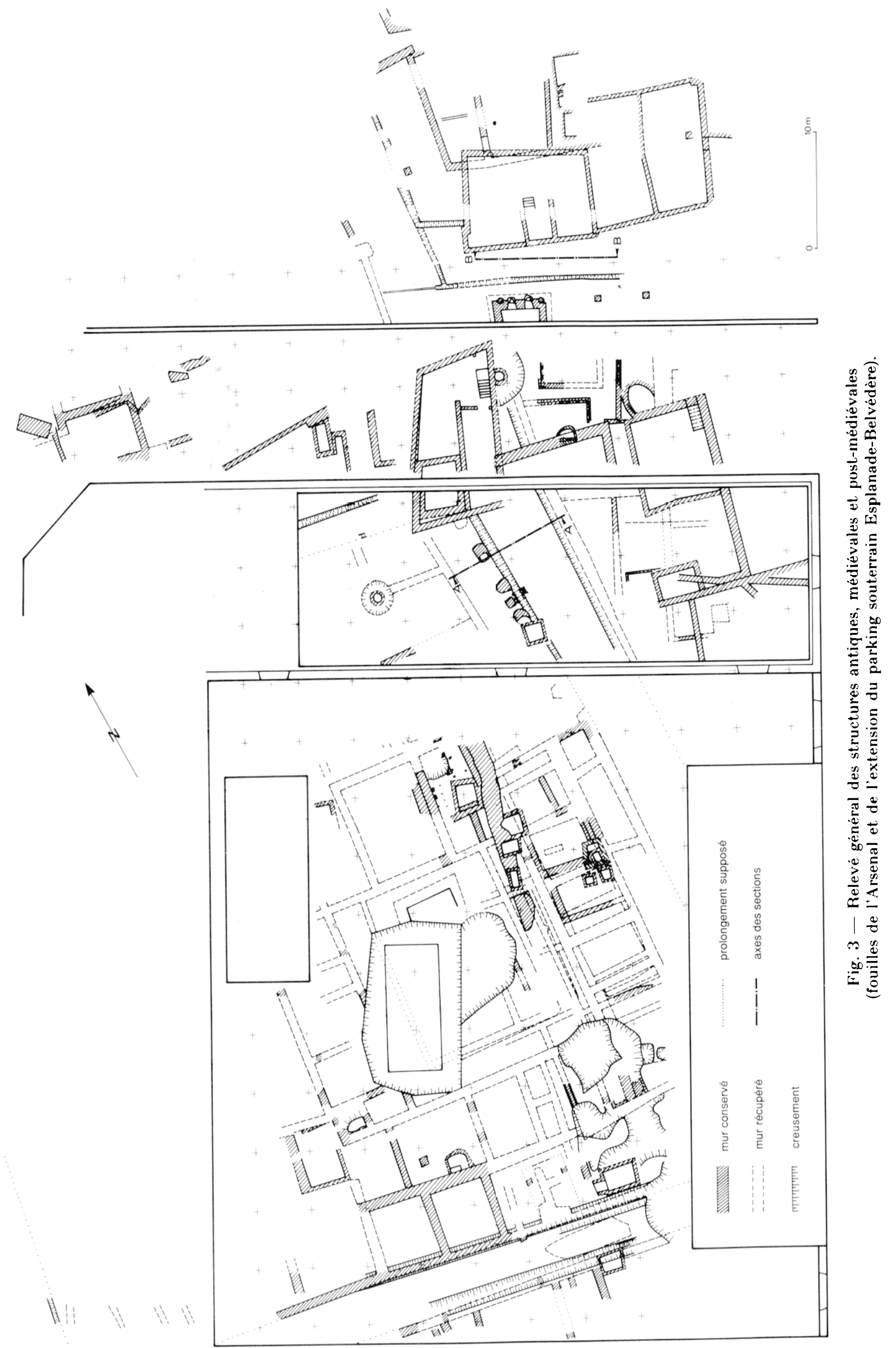


sous la rue Winston-Churchill, entre les Nouvelles Galeries et la galerie marchande, s'est déroulée de juin à juillet 1987 sur une surface de $600 \mathrm{~m}^{2}$ environ. L'extension du parking souterrain Esplanade-Belvédère est à l'origine de la seconde intervention menée en novembre et décembre 1987 sur plus de $1000 \mathrm{~m}^{2}$.

Le secteur du passage souterrain, dont l'organisation et la topographie se rattachent à celles du centre ville actuel, fera l'objet d'une étude séparée. Le chantier de l'Arsenal et celui de l'extension du parking, situé immédiatement au nord, appartiennent au contraire à un même quartier, défini à l'origine par son relief, qui a été considérablement remanié depuis le $\mathrm{XvI}^{\mathrm{e}} \mathrm{s} .{ }^{16}$. Deux articles relatifs aux fouilles de l'Arsenal sont d'ores et déjà parus, portant sur la céramique et le verre de deux ensembles clos des XIV et XVI ${ }^{e}$ s. (Bourger, Cabart, 1990) et sur un atelier métallurgique de la seconde moitié du III $^{\circ}$ s. (Leroy el alii, 1990). Le présent travail est exclusivement consacré à la topographie urbaine et à l'évolution de l'habitat du quartier durant l'Antiquité ; le mobilier archéologique, qui fera prochainement l'objet d'un mémoire de maitrise, n'est évoqué ici qu'en tant qu'élément de datation.

\section{ÉTAT DES CONNAISSANCES}

Le quartier est situé à l'ouest du plateau d'interfluve dessiné dans les terrasses du Würm par les lits de la Moselle et de la Seille. Il occupe une petite éminence d'environ $183 \mathrm{~m}$ d'altitude dominant la Moselle de $15 \mathrm{~m}$ et séparée de la colline de SainteCroix par un ensellement peu profond (Lefebvre, Wagner, 1984, p. 152).

Dès le $\mathrm{XIX}^{\mathrm{e}} \mathrm{s}$, plusieurs découvertes fortuites révèlent la présence dans ce secteur de la ville de vestiges gallo-romains importants (Keune, 1900, p. $346,349,353$ et 1904, p. 478 ). Le site, compris dans le périmètre défini par l'enceinte du BasEmpire, est proche de Saint-Pierre-aux-Nonnains (fig. 4). Les fouilles de grande envergure effectuées en

16 A partir de 1562, la construction de la Citadelle s'accompagne de travaux considérables (démolition de 250 maisons, établissement de fortifications bastionnées et de fossés). Au XIX ${ }^{\mathrm{C}}$ s., le secteur connaît à nouveau d'importants nivellements, nécessités par l'aménagement de l'Esplanade, puis la réalisation de la cour et des bâtiments de larsenal Ney. De cette période date la restructuration du parcellaire et l'implantation d'artères nord-est/sud-ouest et nord-ouest/sud-est.

Ces creusements et nivellements successifs expliquent l'important arasement des niveaux de l'Antiquité tardive et surtout du Moyen Age, dont ne subsistent que des structures excavées.
1942 par W. Reusch (1943a) ont démontré la réutilisation dans cet édifice médiéval d'une construction à plan basilical considérée aujourd'hui comme appartenant à un ensemble thermal de la fin du ives. (Delestre, 1988a, p. 27;1988b). Sous ce bâtiment, la découverte d'un four de potier du $\mathrm{I}^{\mathrm{er}} \mathrm{s}$. indique que le site fut occupé dès le Haut-Empire (Reusch, $1943 \mathrm{~b} ; 1944)$. Les recherches reprennent en 1958 au nord-ouest du monument sous la direction de J.-J. Hatt (1960, p. 217-219; 1961；1962, p. 494496); plusieurs bassins ainsi que des fondations appartenant à l'époque gallo-romaine sont partiellement dégagés. Quelques années plus tard, lors des travaux d'agrandissement de l'École des arts appliqués, attenante à Saint-Pierre-aux-Nonnains, des murs orientés ouest-nord-ouest/est-sud-est et des niveaux de sols superposés révèlent une occupation continue du $\mathrm{I}^{\mathrm{er}}$ au $\mathrm{IV}^{\mathrm{e}} \mathrm{s}$.; la présence d'hypocaustes, d'enduits peints et d'éléments d'architecture de qualité laisse penser que ces habitations étaient loin d'être modestes (Collot, 1967-1968). Malheureusement la fouille s'est déroulée dans des conditions telles qu'il n'a pas été possible de restituer leurs plans.

$\mathrm{Au}$ nord-est, la construction d'un parking souterrain sous l'Esplanade en 1964 entraîne des travaux de terrassement importants. Les observations stratigraphiques effectuées par G. Collot et J.-J. Hatt (Collot, 1964 ; Hatt, 1964) montrent que le quartier était fréquenté dès le début du $\mathrm{I}^{\mathrm{er}} \mathrm{s}$. L'occupation présente globalement les mêmes caractéristiques que celle mise en évidence sur le chantier de l'École des arts appliqués et semble se poursuivre également durant l'Antiquité tardive. Une rue orientée nord-ouest/sud-est a été découverte; cet axe est conforme aux orientations du quadrillage supposé du centre de la ville.

Les perspectives de recherche développées lors des fouilles récentes se sont donc nourries des résultats accumulés depuis près d'un siècle, à l'occasion d'interventions archéologiques d'emprise souvent limitée. Si la chronologie de l'occupation était d'ores et déjà acquise dans ses grandes lignes, l'ouverture de surfaces importantes constituait une bonne opportunité pour préciser l'organisation du quartier. En effet, l'orientation de Saint-Pierre-aux-Nonnains et d'un certain nombre de vestiges, distincte de celle du quadrillage envisagé pour le centre de la ville, laissait soupçonner la coexistence ou la succession d'au moins deux trames urbaines (Jolin, 1977, p. 25, note 8 ). Plusieurs explications avaient alors été proposées (influence du relief, du tracé de la voie de Scarponne, ou de l'enceinte...), qu'il convenait de vérifier. 


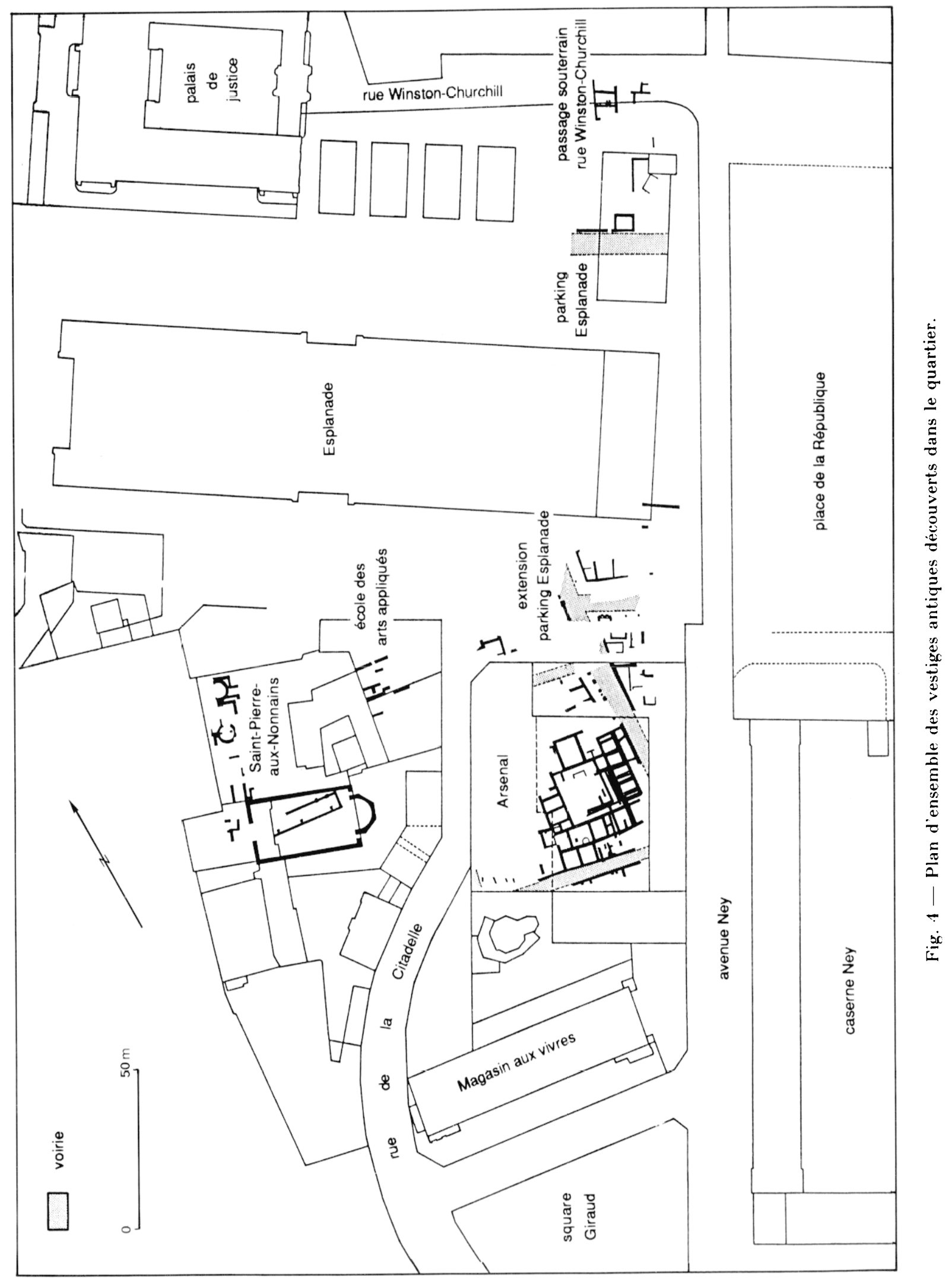


Par ailleurs, en dépit de nombreuses observations ponctuelles, l'habitat n'avait pu jusqu'alors faire l'objet de reconnaissances extensives. Parmi les thèmes de recherche prioritaires, figuraient donc l'agencement des maisons d'un îlot urbain, leurs techniques de construction, l'évolution du parcellaire.

Les travaux récents ont permis de comprendre l'organisation du secteur étudié, mais également de réenvisager la topographie urbaine à l'échelle du quartier. Le système de voirie découvert à cette occasion constitue l'un des apports les plus novateurs de ces fouilles.

\section{LA VOIRIE}

Cinq nouvelles rues, qui définissent plusieurs ilots, ont été partiellement reconnues (fig. 5). Si l'on excepte des modifications mineures de largeur, liées éventuellement à l'empiètement du bâti sur la rue, ce schéma, implanté dès la première moitié du $\mathrm{I}^{\mathrm{er}} \mathrm{s}$., ne semble pas connaître d'évolution importante jusqu'à la fin de l'Antiquité. Toutefois, en raison de la pauvreté des éléments de datation issus des couches de cailloutis ou des radiers, il n'a pas été possible de dater précisément les états successifs des chaussées.

\section{LA RUE 1}

Orientée sensiblement nord-sud, elle semble avoir joué un rôle primordial dans l'organisation du quartier. Elle se situe en effet au contact de deux réseaux de voirie d'orientations différentes. Sa largeur moyenne de l'ordre de $5 \mathrm{~m}$, la présence d'un trottoir puis d'un portique à l'ouest (îlot B) et d'un second portique à l'est (îlot E) confirment son importance.

Cette rue a été principalement étudiée lors des fouilles de l'Arsenal, où une tranchée de sondage a permis de reconnaitre cinq niveaux d'utilisation (fig. 3 et 6A). Les chaussées, à l'exception de la plus ancienne, présentent un bombement caractéristique et sont flanquées de caniveaux. Le premier sol de cailloutis est installé directement sur le paléosol; en revanche, les suivants reposent sur de puissants radiers, constitués de blocs de calcaire encastrés, disposés de chant. La rue du deuxième état est bordée le long de l'îlot $\mathrm{B}$ d'un caniveau en $\mathrm{V}$ et d'un trottoir large de $3,20 \mathrm{~m}$ la dominant légèrement. Pour le troisième état, la rue, munie de caniveaux de chaque côté, est décalée vers l'ouest mais conserve le même trottoir. Le déplacement des caniveaux des deux derniers états conservés provoque un rétrécissement de la chaussée dont la largeur passe de $6 \mathrm{~m}$ à $4,50 \mathrm{~m}$. Le caniveau qui longe le trottoir est aménagé à l'aide

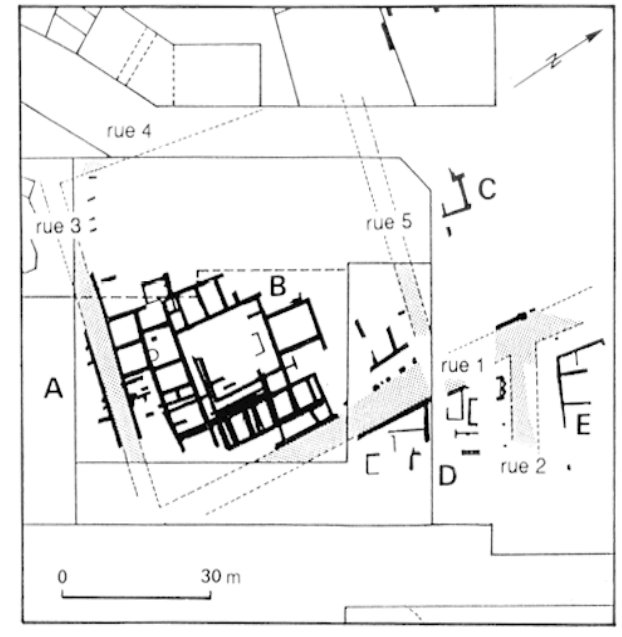

Fig. 5 - . Nomenclature du secteur de l'Arsenal.

de planches ou de piquets en bois et de dalles verticales en pierre. Le portique ouest atteint une longueur de $22 \mathrm{~m}$ environ. Ses piliers reposaient vraisemblablement sur des dés en pierre dont ne subsistent que les fondations.

Dans un premier temps, le trottoir se prolongeait sans doute au sud. Toutefois, dès la seconde moitie $\mathrm{du} \mathrm{I}^{\text {er }} \mathrm{s}$., les constructions empiètent sur cet espace de circulation et s'étendent alors jusqu'à la rue (cf. infra, p. 21). A sa jonction avec la rue 5 , la rue 1 s'élargit légèrement, sans doute pour faciliter les manœuvres des véhicules. Au cours du chantier d'extension du parking, l'observation d'un caniveau creusé dans le sable naturel a permis de supposer une largeur initiale d'au moins $7,50 \mathrm{~m}$ pour la portion de la rue 1 au nord du carrefour avec la rue 2 .

\section{$L A R U E 2$}

De direction nord-ouest/sud-est, elle suit les orientations supposées pour le centre de la ville et présente une déclivité de $12 \%$ (fig. 3 et $6 \mathrm{~B}$ ). Sa chaussée de $5 \mathrm{~m}$ de largeur est bordée de part et d'autre par un caniveau dont l'emplacement n'a pas beaucoup varié au cours du temps. La découverte d'une frette en fer d'environ $15 \mathrm{~cm}$ de diamètre dans le prolongement du caniveau sud indique qu'une conduite enterrée en bois devait relier le fossé ouest de la rue 1 au fossé sud de la rue 2 .

A peu de chose près la structure de la rue 2 est identique à celle de la précédente. Un premier niveau de circulation constitué de graviers fins est installé sur le sol naturel; lui succèdent au moins cinq recharges, parfois simplement réalisées avec des cailloutis compacțés mais qui comportent dans trois cas un véritable radier de pierres calcaires posées de chant. 

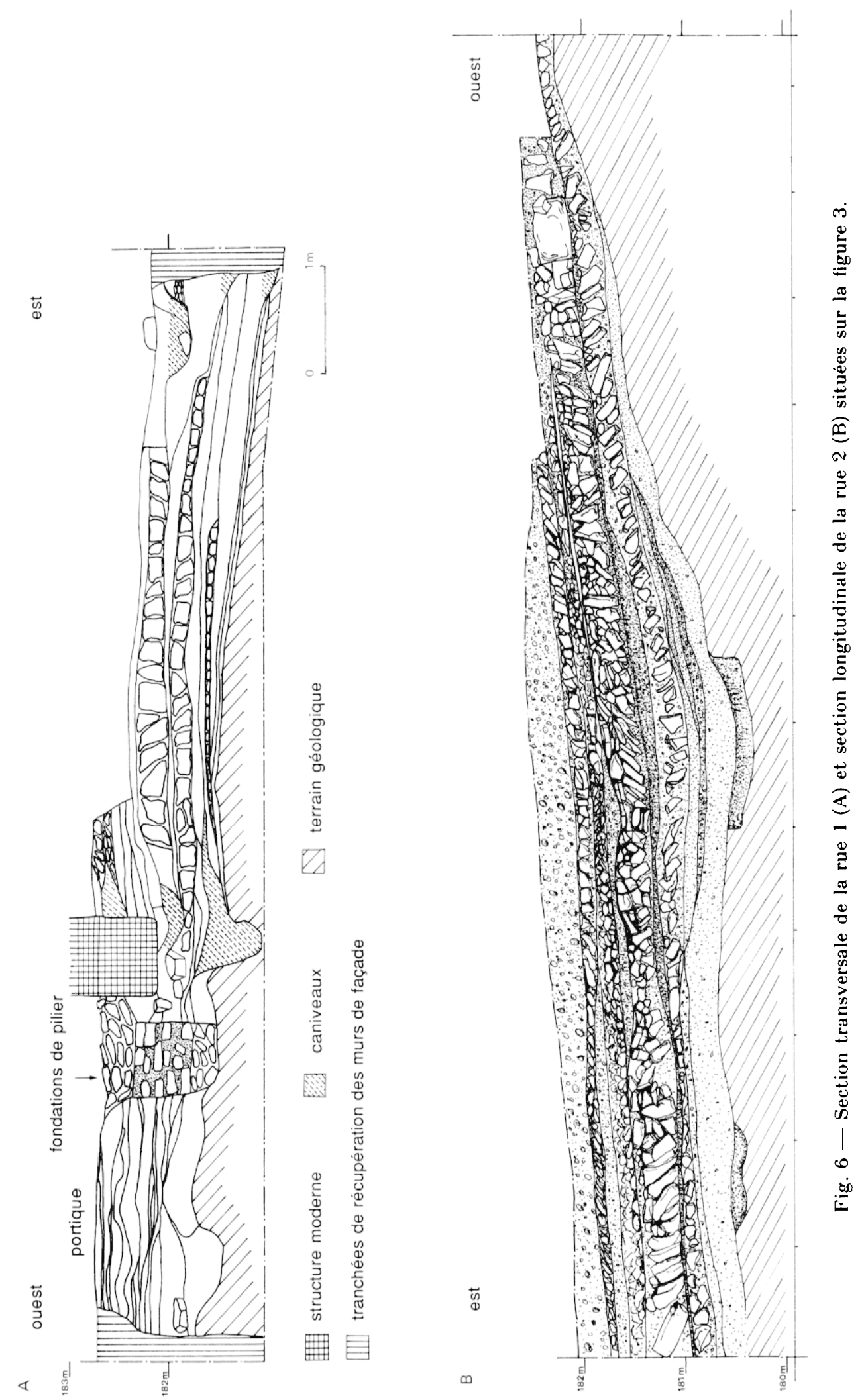
Un portique, dont les piliers reposaient sur des dés de pierre calcaire d'environ $0,60 \mathrm{~m}$ de côté. longe la rue au sud, tandis quau nord, un trottoir de $4,20 \mathrm{~m}$ de large borde le mur de façade.

\section{LA RUE 3}

Orientée ouest - nord-ouest/est - sud-est, la chaussée n'est conservée que sur $8 \mathrm{~m}$ de longueur; les fondations des murs de façade ont permis cependant de suivre son tracé sur près de $35 \mathrm{~m}$ (fig. 7). En forte déclivité $(13 \%)$, la rue est beaucoup moins large que les rues 1 et $2(2,80 \mathrm{~m})$. Quatre niveaux de circulation ont été définis. Le plus ancien, constitué de cailloutis, est directement installé sur le sol naturel. Les deuxième et troisième sols, en gravier damé, sont renforcés sur les côtés par des pierres plates (fig. 8). Enfin, une recharge de cailloutis a sans doute compensé un affaissement localisé. Deux caniveaux bordent la rue de part et d'autre. Les murs de façade conservés se superposent au système de caniveaux le plus ancien, ce qui manifeste, comme pour la rue 1, un empiètement sur le domaine public.

\section{LA RUE 4}

Située dans un secteur particulièrement arasé, sa présence n'est attestée que par son fossé est. Il est donc impossible d'estimer sa largeur. En revanche, on peut admettre que son tracé est sensiblement perpendiculaire à la rue 3 .

\section{LA RUE 5}

De même orientation que la rue 3 , elle est un peu moins large. Sa chaussée est repérée sur $6 \mathrm{~m}$ de long et matérialisée par un lit de gravier compacté, posé sur le sol naturel. Un seul fossé la borde au nord. Cette rue ne se prolonge pas au-delà de sa jonction avec la rue 1 .

Dans leur structure, ces rues sont tout à fait comparables à nombre de celles qui ont été observécs en Gaule. Comme le soulignent les études récentes, les chaussées étaient en effet rarement dallées, que ce soit à Amiens (Bayard, Massy, 1983, p. 59), Saintes (Lauranceau, Maurin, 1988, p. 25-26), Narbonne (Sabrié, Solier, 1987, p. 48-53) ou bien sur des sites plus proches tels Bliesbruck (Petit, Schaub, 1990, p. 131) ou Hettange-Grande (Stachowski, 1990, p. 155). Dans toutes ces agglomérations, les rues étaient fréquemment bordées de trottoirs ou de portiques dont les piliers reposaient, comme à l'Arsenal,

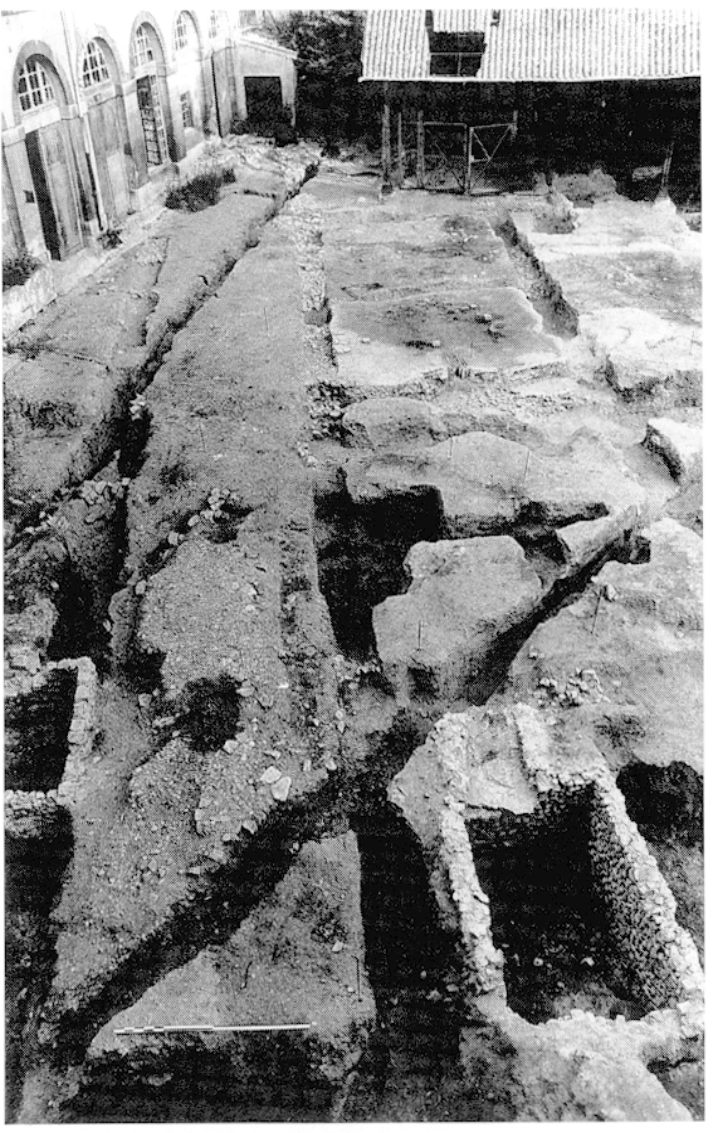

Fig. 7 - Rue 3 vue de l'est.

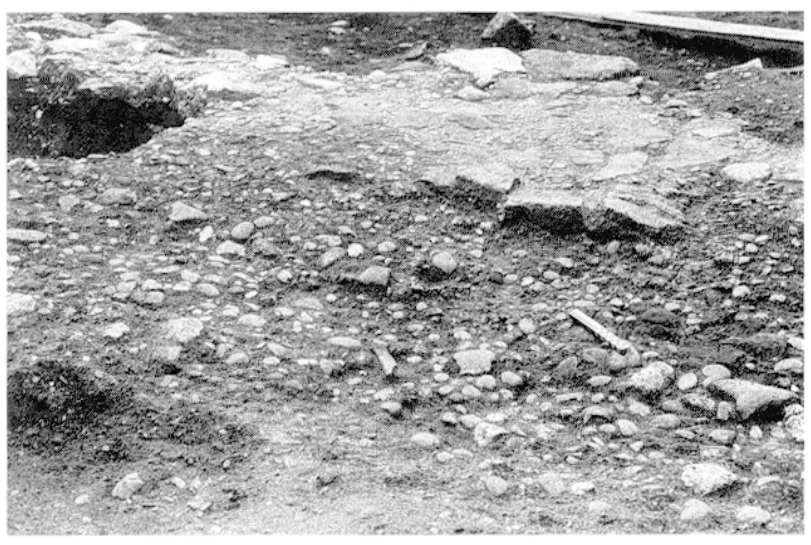

Fig. 8 - Détail du troisième état de la rue 3 .

sur des dés en pierre. En ce qui concerne les caniveaux, les aménagements constitués de planches et de pieux en bois, identifiés ponctuellement le long des rues 1 et 5 , sont comparables aux coffrages mentionnés à Amiens (Bayard, Massy, 1983, p. 60, fig. 16), ou à Rouen (Rouen gallo-romain, 1982, p. 22). 


\section{LES CANIVEAUX : ANALYSE DU REMPLISSAGE}

La mise au jour lors des fouilles de l'Arsenal de quatre rues antiques a suscité le développement d'un programme d'étude des traces organiques présentes dans le remplissage des caniveaux ${ }^{17}$. L'objectif principal de ce travail était de déterminer la nature des effluents évacués, afin de mieux cerner la fonction de ces caniveaux, et indirectement, les pratiques d'hygiène et de gestion des déchets dont ils témoignent. Les prélèvements ont été effectués sur les états les plus anciens des rues 3 (caniveau sud, V21), 4 (caniveau est, V20), 5 (caniveau nord, V11, V11') et des phases plus récentes des rues 3 (caniveau nord, V9) et 1 (caniveau ouest, V10, V10').

La quantité de matière organique retrouvée indique l'utilisation probable de ces caniveaux comme égouts. En revanche, en dépit des conditions de conservation favorables, aucune trace de coprostanol (marqueur moléculaire caractéristique de la matière fécale de l'homme et des animaux supérieurs) n'a été décelée, ce qui prouve qu'ils n'ont pas servi à évacuer des excréments.

L'analyse montre une similitude entre d'une part V9, V10 et $\mathrm{V}^{10}{ }^{\prime}$ (quasi-identité des concentrations totales en acides gras, stérols, et distributions similaires obtenues pour tous les constituants lipidiques) et d'autre part V11, V11' et V20 (particulièrement pour leur analogie dans les répartitions en n-alcanes, acides, stérols et alcools). V21 se distingue des autres échantillons par ses concentrations nettement plus élevées en composés lipidiques. La figure 9, qui présente les pourcentages en stérols d'origine animale par rapport aux stérols totaux et les pourcentages en acides légers (moins de vingt atomes de carbone) par rapport aux monoacides totaux, pour chaque échantillon, illustre ces analogies.

On peut remarquer une certaine corrélation entre ces deux groupes principaux et la datation relative des caniveaux prélevés, mais d'autres critères (topographiques par exemple) ont pu jouer, et l'interprétation se révèle délicate, faute de points de comparaison et compte tenu du nombre insuffisant. de prélèvements.

17 Ces analyses (chimie organique) ont èté effectuées par C. Pepe, dans le cadre d'un doctorat en chimie, sur l'. 1 pplication des marqueurs géochimiques dans l'étude des paléoenvironnements à intérêt archéologique (Paris VI, 1988). La méthode utilisée est brièvement présentée dans le cadre d'une note consacrée à ce programme (Pepe, 1990). Nous remercions très chaleureusement le Laboratoire de physique et chimie marines pour son amicale collaboration.

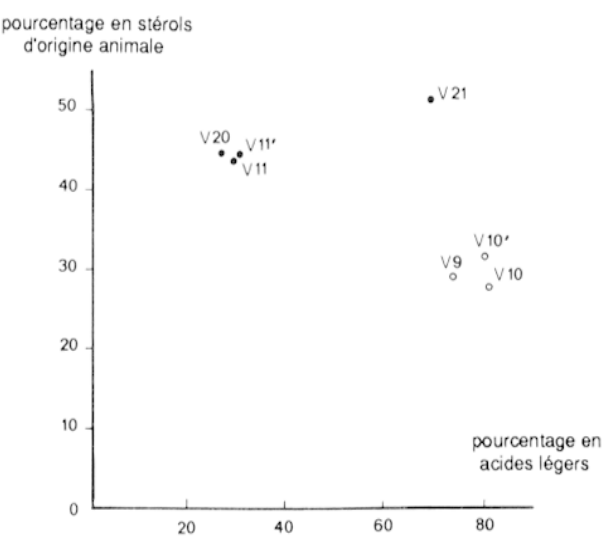

Fïg. 9- Analyse des prélèvements de caniveaux : les échantillons figurés en noir correspondent aux ètats les plus anciens.

Yéanmoins, il est évident que les lipides identifiés sont le résultat de deux types d'apports:

- les apports végétaux/animaux dus à l'écosystème mème du caniveau (recouvert ou nonrecouvert) tels que les organismes vivants, feuilles, branches, etc. Les apports en biomasse des végétaux sont toujours prépondérants ;

- les rejets anthropiques végétaux/animaux.

Au regard des résultats, notamment des proportions en acides lourds, caractéristiques des végètaux supérieurs, il semble que pour V20, V11 et V11' (qui possèdent environ $70 \%$ d'acides lourds par rapport aux acides totaux), les apports de l'écosystème du caniveau dominent. De nombreux facteurs peuvent être évoqués (absence de désherbage et d'entretien du caniveau et de ses abords, toitures en matières végétales, habitudes alimentaires ou domestiques ...) ce qui doit nous inciter à une grande prudence.

Il n'a malheureusement pas été possible d'effectuer des prélèvements complémentaires, mais ces premiers résultats montrent d'ores et déjà tout l'intérèt de ce type d'analyses, qui devraient notamment nous permettre de mieux appréhender la gestion des déchets en milieu urbain.

\section{L'HABITAT AU I I $^{\mathrm{er}} \mathrm{S}$.}

L'ensemble de nos informations relatives au $\mathrm{I}^{\mathrm{er}} \mathrm{s}$. concerne l'îlot $\mathrm{B}$, où l'organisation de l'habitat a été étudiée sur environ $500 \mathrm{~m}^{2}$ en bordure de la rue 1. Les niveaux antérieurs au milieu du $\mathrm{I}^{\mathrm{er}} \mathrm{s}$. n'ont pu cependant faire l'objet d'une reconnaissance extensive, en raison des profondes destructions liées aux reconstructions successives et des délais impartis à la fouille ... 


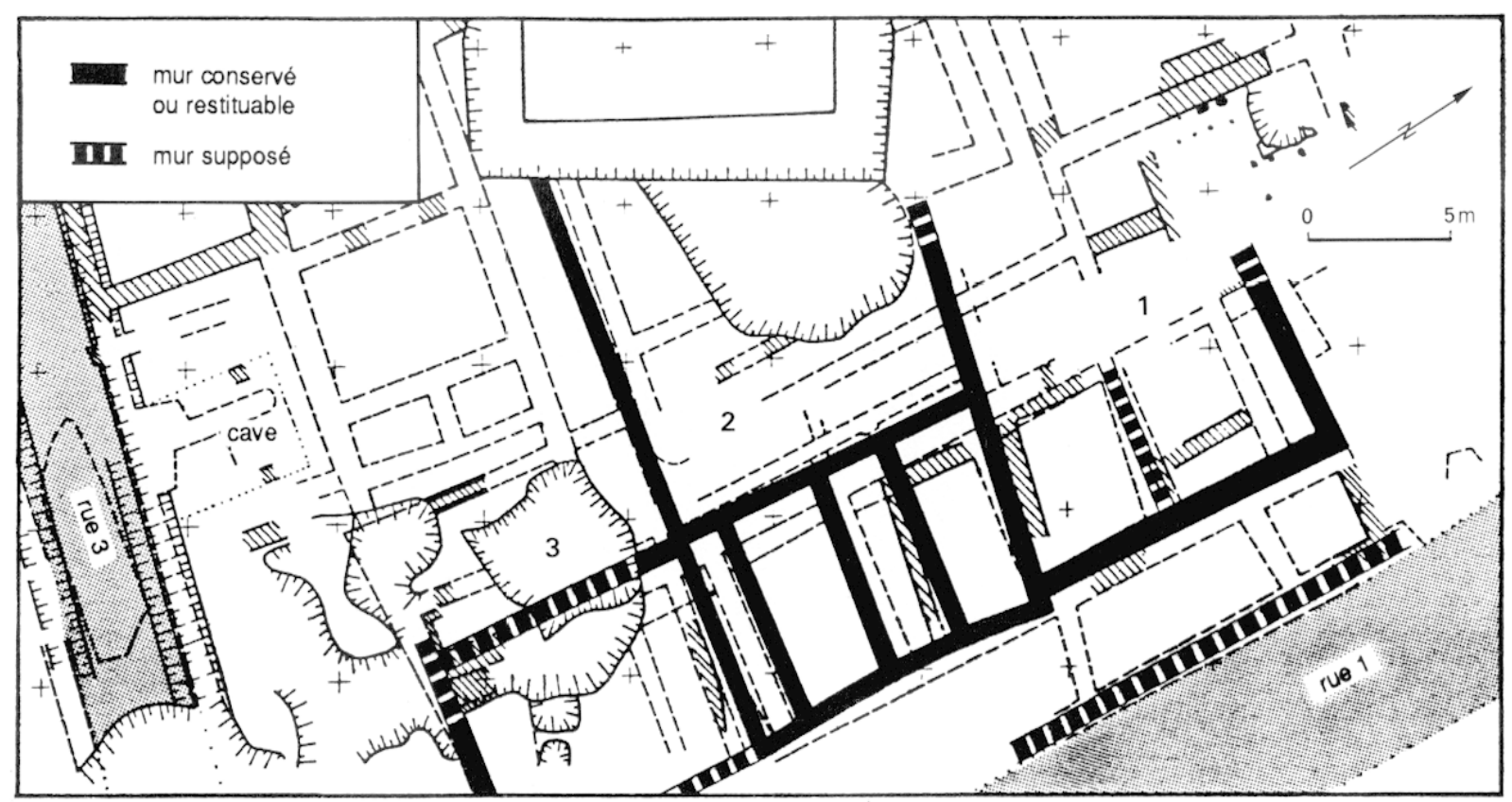

Fig. 10 - État 1B (40-60/70).

\section{LES PREMIËEES OCCUPATIONS (PREMIËRE MOITIÉ DU I Ir S.)}

Le seul habitat identifié avec certitude se trouve au nord de la cour de l'Arsenal. Plusieurs trous de poteaux et piquets alignés selon un axe nord-nordest/sud - sud-ouest ont été reconnus. Le sol en terre battue conservait encore les traces de deux foyers. Il n'a pas été possible de définir les limites de cette construction, détruite en grande partie par l'édification d'habitats postérieurs, mais son orientation est comparable à celle des structures plus tardives. La présence de céramique augusto-tibérienne en d'autres points du site laisse supposer que cette occupation n'était pas isolée. Ce type d'habitat de tradition laténienne a déjà été repéré à Metz, notamment sur le Haut de Sainte-Croix (Brunella et alii, 1988 , p. 28).

\section{ÉTAT $1 B(40-60 / 70)$}

Des unités d'habitation, mesurant 9,50 à $11 \mathrm{~m}$ en façade, sont établies perpendiculairement à la rue 1 , et bordées vraisemblablement par une galerie large de $3,50 \mathrm{~m}$ environ (fig. 10). Les murs mitoyens se prolongent assez profondément en cœur d'îlot, mais les bâtiments sur rue ne dépassaient peut-être pas - pour cet état - une largeur de $7 \mathrm{~m}$.

L'habitation la mieux conservée (fig. $10, \mathrm{n}^{\circ} 2$ ) est divisée en quatre pièces larges de $1,20 \mathrm{~m}, 2,80 \mathrm{~m}$,
$2 \mathrm{~m}$ et '2,80 m, orientées dans l'axe du bâti. Les trois pièces au sud possèdent un sol de terre battue, celui de la dernière pièce est un sol de galets et de tuileau compactés, sans radier. Le sol de la pièce la plus étroite (couloir d'entrée?) se situe $25 \mathrm{~cm}$ plus haut que les autres. La pièce immédiatement au nord comportait un foyer, très partiellement conservé.

De la maison 1, dont la limite ouest n'est pas conservée, seule la pièce nord a pu ètre étudiée. Son sol en cailloutis et tuileau repose sur le paléosol; sa construction a nécessité le rattrapage du dénivelé $d u$ terrain naturel par l'apport d'un remblai du côté est.

Les deux limites latérales du bâtiment 3 sont précisément connues, mais il ne semble pas qu'une cloison interne ouest-est ait existé. Trois sols successifs en terre battue ont été fouillés, dont le ou les plus récents pourraient appartenir à l'état suivant. Sur chacun d'entre eux est établi un foyer monté à l'aide de fragments de tuiles liés à l'argile, construit chaque fois à un emplacement différent. Cette zone est très perturbée par l'implantation d'une grande fosse de prélèvement de sable à l'époque moderne ( $\mathrm{XVI}^{\mathrm{e}} \mathrm{s}$.).

Les données concernant l'architecture sont assez modestes en raison de la récupération presque totale des murs lors de l'implantation de l'état 1C. Toutefois, les matériaux non réutilisés ont alors servi de remblai et indiquent l'emploi de limon et de briques crues en argile. Sur le sol de galets compactés de l'habitat central, se trouvaient ainsi une vingtaine de briques en argile, pour la plupart d'un module de 


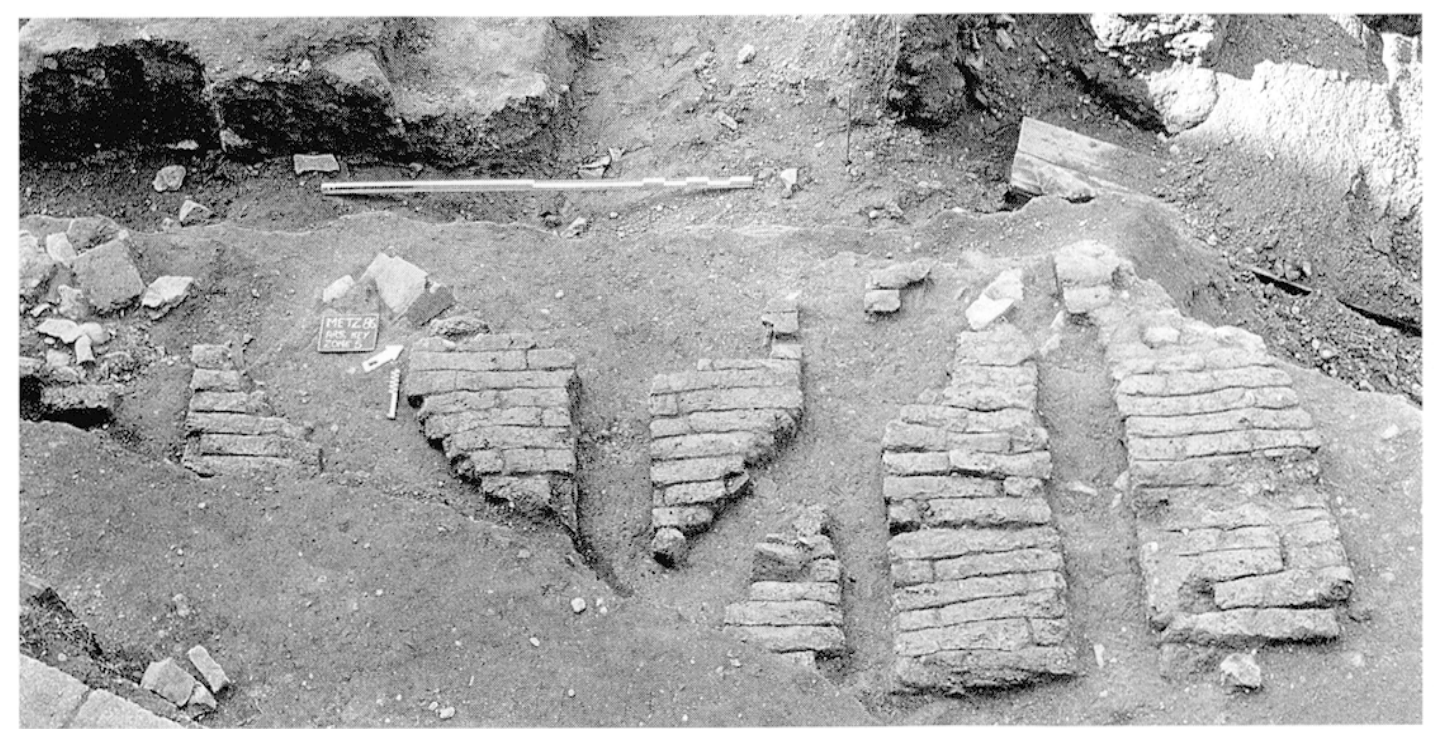

Fig. 11 - État 1B. Mur à pan de bois et briques crues, effondré à plat. La base du mur est située en bordure supérieure du cliché.

$22 \times 12 \times 8 \mathrm{~cm}$. Effondrée sur le sol de la galerie en façade de cette même maison, une cloison en pan de bois hourdi de briques crues a pu également être observée (fig. 11). Longue de $5 \mathrm{~m}$ et haute de $2,20 \mathrm{~m}$ au minimum, elle limitait à l'est les deux pièces centrales, sans fermer la plus étroite, ce qui semble confirmer la vocation de couloir d'entrée de cette dernière. L'ossature est constituée de neuf poutres de section quadrangulaire, de $0,30 \mathrm{~m}$ de largeur maximale et $0,10 \mathrm{~m}$ d'épaisseur, dont subsiste l'empreinte. Sept sont placées verticalement, espacées tous les $0,70 \mathrm{~m}$ et deux autres, disposées obliquement à $45^{\circ}$ de part et d'autre de la poutre centrale, les relient entre elles. Les briques en argile, d'un module assez régulier ( $44 \times 10 \times 10 \mathrm{~cm}$ environ) présentent des joints épais de $2 \mathrm{~cm}$, en limon argileux. Aucune trace de revêtement sur l'une ou l'autre des faces n'était conservée. Les poutres verticales devaient être fichées dans une sablière horizontale qui a disparu. La destruction semble volontaire, liée à la récupération d'un soubassement maçonné, dont témoignent les vestiges de la fondation en pierre, profonde de 0,50 à $0,60 \mathrm{~m}$. L'ensemble des poutres a été récupéré puisque leur emplacement dans la cloison effondrée est soigneusement remblayé.

Les fragments de peintures murales retrouvés dans le niveau de destruction de l'habitat central, ornés de mouchetis rouge, vert, jaune sur fond crème ou rosé, ou bien de motifs géométriques verts sur fond rose, fournissent quelques indications sur le décor de cette maison.
De la galerie en façade supposée ne sont conservés que des niveaux de sol en terre battue régulièrement rechargés; de profondes tranchées de récupération nous interdisent toute restitution des supports et du couvrement. La hauteur probable, de l'ordre de $3 \mathrm{~m}$, du pan de mur effondré la limitant à l'ouest est en tout cas suffisante pour permettre l'accrochage d'une toiture inclinée à $20^{\circ}$ environ, sans entraver les possibilités de circulation dans la galerie.

Le matériel associé à l'occupation est relativement abondant : céramique, vestiges osseux, quelques éléments métalliques. Les principales catégories de céramique représentées illustrent les différents aspects de la vie domestique : dolia et quelques tessons d'amphores, pots en céramique commune grise, cruches en pâte claire engobées ou non, vases-tonnelets ou assiettes en gallo-belge et quelques tessons de sigillée de Gaule du Sud (fig. 13, nos 1-4). L'ensemble permet de dater assez précisément cet état des années 40 à $60 / 70$.

\section{ÉTAT IC (VERS 60/70)}

Les maisons reprennent les mêmes limites parcellaires, mais légèrement décalées, les murs mitoyens étant en effet reconstruits tout contre leurs prédécesseurs (fig. 12). Seul, le mur séparant les parcelles 2 et 3 conserve le mème tracé dans sa portion ouest, ce qui semble confirmer que cette portion n'était pas pour l'état 1B comprise dans les corps de bâtiments proprement dits. Ce décalage devait facili- 


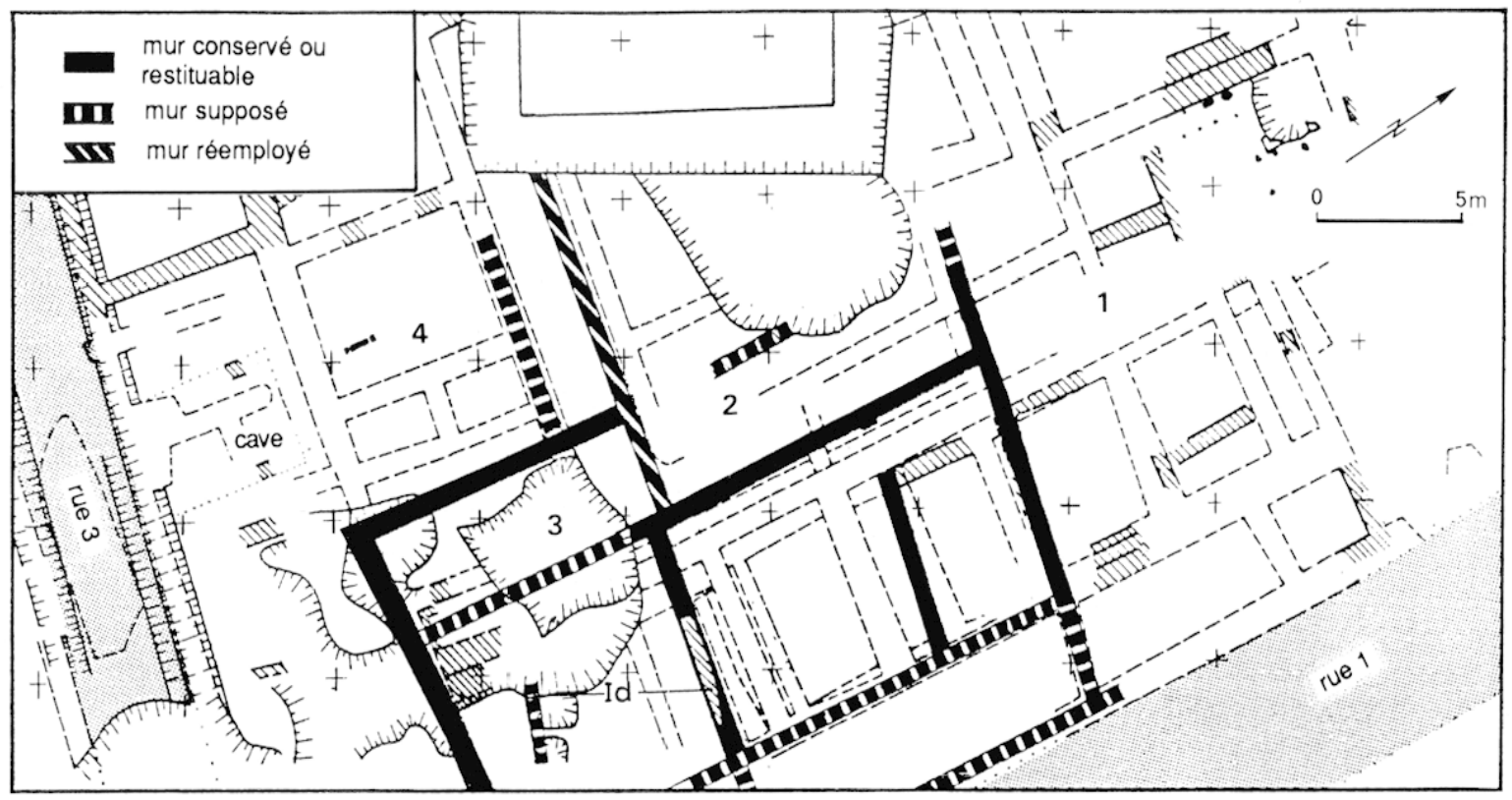

Fig. 12 - État 1C (vers $60 / 70)$.

ter l'échelonnement des phases de construction et de démolition : il était ainsi possible de reconstruire entièrement les bâtiments 1 et 3 sans avoir à intervenir sur le bâtiment central. La démolition des maisons de l'état précédent est marquée par la récupération systématique de matériaux : pierres des solins, sablières basses et poutrage des cloisons à pan de bois... Les déblais non récupérables, nivelés, ont servi de remblai d'installation aux nouveaux sols.

Les reconstructions s'accompagnent sans doute d'un accroissement des surfaces bâties. Ainsi, la maison 3 voit sa surface augmenter de moitié par l'ajout à l'arrière d'une pièce large de $3 \mathrm{~m}$, et une disposition identique est possible pour la maison 2. De plus, la tranchée de récupération qui prolonge le mur mitoyen $1 / 2$ vers l'est permet d'envisager une fermeture du portique, et donc la création en façade de la maison 2 d'une pièce allongée de $11,50 \times 3 \mathrm{~m}$.

Au sud, une unité d'habitation 4 a été partiellement étudiée. Elle est mitoyenne à l'est avec le bâtiment 3, mais ne sc prolonge pas vers le nord jusqu'au mur 2/3. Deux sols en terre battue successifs, pourvus chacun d'un foyer de briques, étaient conservés. La construction, au moins partiellement en torchis, possédait une couverture en tuiles.

Trois fondations en pierre sont encore en place dans le secteur: le mur mitoyen $3 / 4$, une cloison interne ouest-est de la maison 2 et son mur nord. Ce dernier est fondé à $1 \mathrm{~m}$ de profondeur environ et sa partie supérieure bien nivelée témoigne d'une probable élévation en matériaux légers sur sablière basse. La reconstruction de l'habitat 1 apparait vraisemblable, mais l'ampleur des perturbations n'a pas permis d'en retrouver les limites.

Les travaux de nivellement du XIXe s. n'ont en général préservé que le ou les niveaux de sol les plus anciens de l'état $1 \mathrm{C}$. De plus, mis à part la céramique associée au second foyer de la maison 4 (fig. 13, $\mathrm{n}^{\mathrm{os}}$ 5-8), le mobilier demeure peu abondant. Il n'est donc pas possible de préciser la durée d'utilisation de cet état.

\section{ÉTAT ID (FIN DU I I S.?)}

La réédification du mur mitoyen $2 / 3$, implantée sur la récupération de la paroi de l'état $1 \mathrm{C}$, témoigne vraisemblablement d'une troisième phase de travaux, dont l'ampleur nous échappe (fig. 12).

A cet état $1 \mathrm{D}$ est associée une cloison interne du bâtiment 3 établie sur un solin de pierres de $0,25 \mathrm{~m}$ de hauteur, et dont la construction s'est accompagnée d'un arasement important des niveaux antérieurs. Le remblai de sol limoneux déborde sur le solin de $0,10 \mathrm{~m}$ de chaque côté, ce qui indique une élévation plus étroite, sans doute en matériaux légers.

La technique utilisée pour le mur mitoyen apparaît singulière : celui-ci est constitué de deux parements de limon et d'un bourrage central (sédiments sableux et gravats) (fig. 14). On observe entre les parois et le bourrage une limite verticale de couleur sombre qui n'est visiblement pas un clayonnage, 


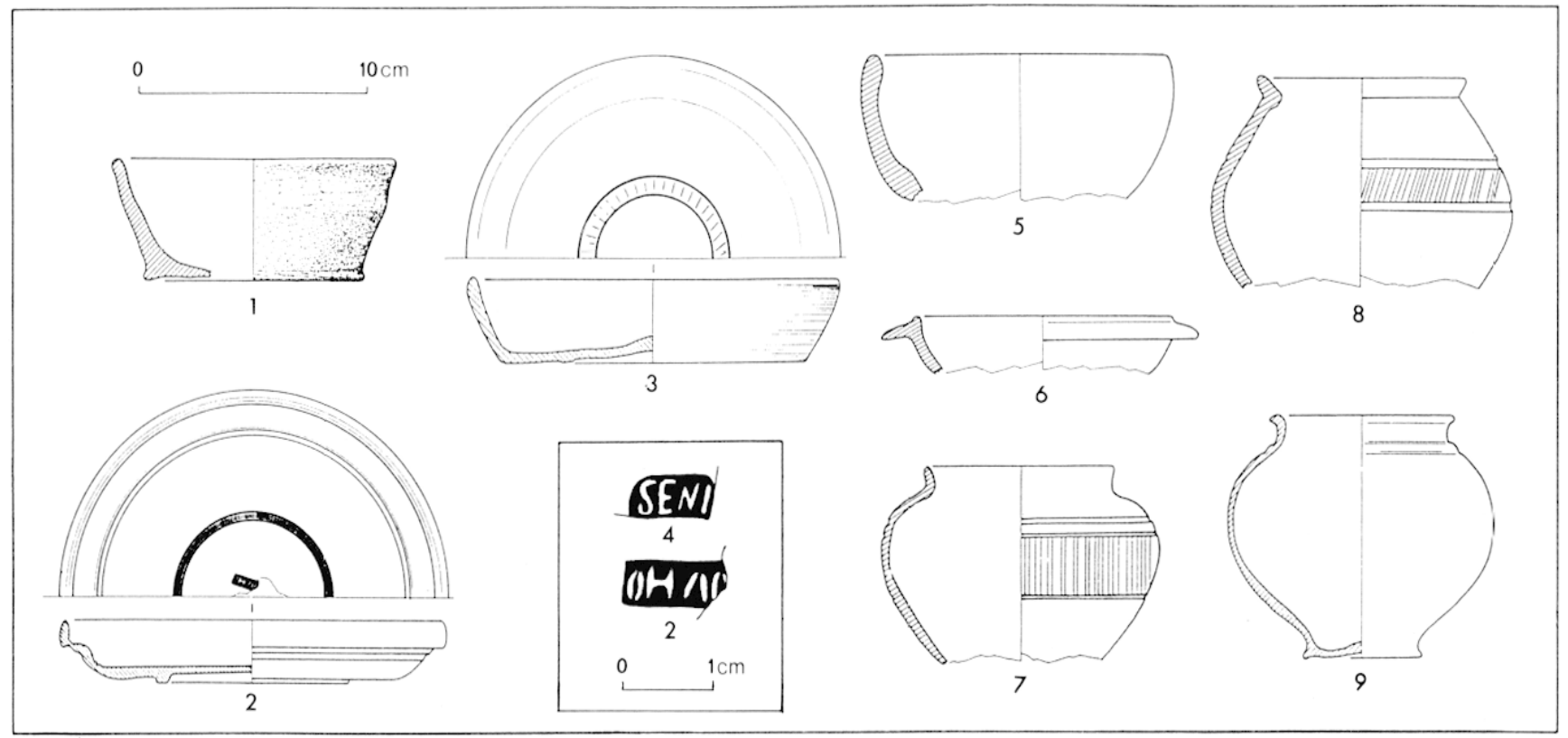

Fig. 13 - Échantillonnage de la céramique provenant des niveaux d'occupation des états 1B et 1C. État $1 B: 1$, commune à pâte noire; 2 , terra nigra; 3 , gallo-belge grise 4 , sigillée de Gaule du Sud. Élat $1 C: 5$. commune à pàte noire: $6-9$, gallo-belge grise.

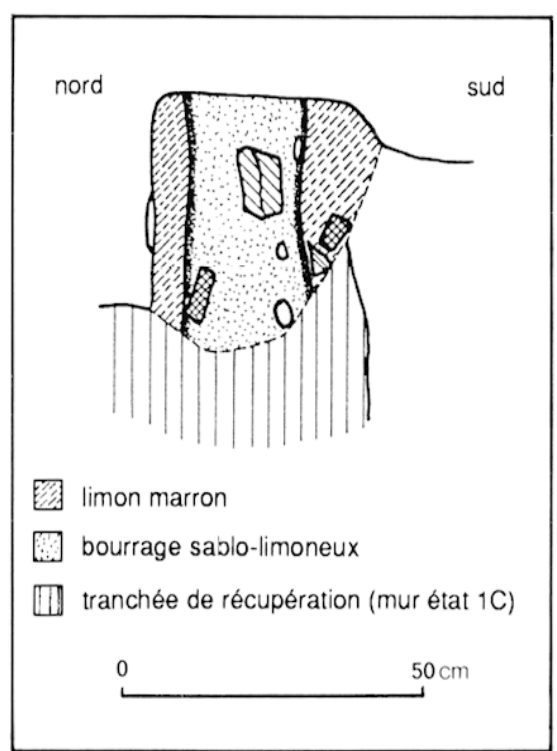

Fig. 14 - État 1D. Section du mur mitoyen $2 / 3$.

mais pourrait correspondre par exemple à l'empreinte de planches disparues.

Compte tenu de l'absence presque totale de mobilier, ces travaux ne peuvent être datés précisément; si l'on suppose une durée de l'ordre de 10 à 30 ans pour l'état précédent, l'état $1 \mathrm{D}$ pourrait se situer vers la fin du $\mathrm{I}^{\mathrm{er}} \mathrm{s}$.
En définitive, l'habitat à l'ouest de la rue 1 ne subit pas d'èvolution majeure au cours de la seconde moitie du $\mathrm{I}^{\mathrm{er}} \mathrm{s}$., alors que ces maisons connaissent vraisemblablement deux importantes phases de reconstruction successives. La permanence du parcellaire indique l'existence de lots distincts, larges de $10 \mathrm{~m}$ environ et profonds d'au moins $20 \mathrm{~m}$, qui sont définis dès le milieu du $\mathrm{I}^{\text {er }} \mathrm{s}$. Même si ces données ne concernent qu'un secteur limité, ce schéma est sans doute applicable à une grande partie de l'îlot $B$ et l'urbanisation ne semble pas limitée à une étroite bande de terrain en bordure de la rue la plus importante, comme le montrent des observations effectuées sur les deux rives de la rue 3 .

L'organisation interne des habitats est relativement classique, proche par exemple de celle observée pour le quartier de commerçants et d'artisans d'Alésia (Mangin, 1981, voir notamment l'insula F (est), côté rue 2 - Horizons II la el IIIb, p. 97-113 et pl. 39b et 40a). L'évolution décelée entre les états $1 \mathrm{~B}$ et $1 \mathrm{C}$ (augmentation de la surface construite vers le coeur de l'îlot et possible fermeture de la galerie ou du portique) trouve du reste des parallèles sur le site bourguignon (Mangin, 1981, insula $\mathrm{H}$ et particulièrement ensemble XIXb, p. 125-130 et pl. 57). L'état IC peut être également comparé au "lotissement" étudié à Évreux, rue Saint-Louis, dont certaines habitations sont de surfaces et de plans similaires (Carré, Gerber, 1990, fig. p. 95, bâtiments 1 et 2). 
Les habitats étudiés ne semblent pas dotés de caves, si l'on excepte celle, située en bordure de la rue 3 , quin sondage ponctuel a permis de dater du $1^{r}$ s.. mais qui ne peut ètre rattachée à un état précis.

Les récupérations systématiques et surtout l'ampleur des arasements à l'ouest n'autorisent cependant qu'une vision tronquée de ces habitats; le cocur de l'ilot, notamment, parait relativement vide, à l'exception de quelques fosses-dépotoirs, mais les destructions, auxquelles s'ajoute l'importante surface oblitérée par une citerne moderne, peuvent expliquer la disparition dautres bàtiments, surtout $\therefore$ il sagit de constructions plus légères.

\section{L'HABITAT AUX II $^{\mathrm{e}}-\mathrm{III}^{\mathrm{e}} \mathrm{S}$.}

L'habitat est mieux connu pour les II $^{\mathrm{P}}-\mathrm{III}^{\mathrm{P}} \mathrm{s}$., à l'échelle du quartier. Les travaux récents ont principalement porté sur l'îlot B, occupé en grande partie par une vaste domus d'inspiration romaine, et sur les îlots I) et E, où plusieurs habitations ont pu être partiellement reconnues, à proximité du carrefour de la rue 1 avec la rue 2 (fig. 15 ).

\section{L'ÎLOT B}

I. organisation d'ensemble du secteur sud-est de l'îlot est sensiblement modifiée au II"s. Les trois parcelles précédemment étudiées sont en effet regroupées et englobées dans une propriété plus grande, couvrant près du tiers de la surface totale de l'îlot, et entièrement occupée par une vaste domus.

Cette propriété présente la forme d'un trapèze appuyé d une part sur la rue 1 à l'est et d'autre part sur des unités d'habitation préexistantes au sud, ce qui explique le caractère non rectiligne de sa limite méridionale (fig. 16). A l'ouest et au nord, en revanche, l'ensemble est limité en grande partie par deux murs tirés au cordeau et rigoureusement perpendiculaires $\left(89,5^{\circ}\right)$. Les dimensions de ce quadrilatèré sont remarquables : de parement extérieur à parement extérieur restitués, les côtés nord, ouest, est et sud mesurent respectivement $35,60 \mathrm{~m}$, $35,10 \mathrm{~m}, 35,60 \mathrm{~m}$ et $38 \mathrm{~m}$. Si l'on met à part le côté sud, qui rattrape la légère différence d'orientation que présente l'ensemble par rapport à la rue 1, la moyenne des trois autres còtés est de $35,40 \mathrm{~m}$, avec une variation qui ne dépasse pas $0,8 \%$. Cette mesure, transcrite en pes monelalis $(0,296 \mathrm{~m})$, est de 119.6 p.m.: il s'agit donc manifestement d'une parcelle de $1 \times 1$ actus de 120 p.m.
L'utilisation de l'actus calculé en p.m. lors de limplantation de bàtiments privés est largement attestée en Grande-Bretagne (Walthew, 1978, p. 336-341). Dans un certain nombre de cas, ces habitations sont de dimensions tout à fait comparables à celles de la demeure de l'Arsenal. Ainsi, à Caerwent, la maison II, S mesure $35,80 \times 53 \mathrm{~m}$, soit 1 actus $\times 1, \bar{j}$ actus, et la maison VII, S pourrait occuper une parcelle de $35,70 \mathrm{~m}$ de façade; à Wroxeter, la domus du site VI et la maison 2 de l'insula $\mathrm{XXI}$ mesurent respectivement $35,30 \mathrm{~m}$ et environ $35,80 \mathrm{~m}$ de façade; enfin, à Silchester, le côté ouest de linsula XXVII comprend trois maisons de $35,40 \mathrm{~m}, 36,60 \mathrm{~m}$ et $35,40 \mathrm{~m}$ de façade, et la maison I de l'insula XXXIV mesure 35,20 m (côté nord) sur $37,30 \mathrm{~m}$ (còté ouest) (Walthew, 1978, p. 336, 339$341)$.

La demeure de l'Arsenal s'organise autour d'une cour centrale bordée sur trois côtés par une galerie. L.'ensemble cour-galerie, légèrement losangé, est également de dimensions régulières : $18 \mathrm{~m}$ còté nord, $18,20 \mathrm{~m}$ à l'ouest, $18,10 \mathrm{~m}$ à l'est et $18,10 \mathrm{~m}$ au sud, soit une moyenne de $18,10 \mathrm{~m}$, ce qui correspond à 61 p.m. Ce choix d'un espace central de forme géométrique parfaite, parallèle à la rue 1 , explique la forme légèrement trapézoïdale du corps de bâtiment ouest, établi le long de la limite de propriété. Visiblement, le souci de l' effet architectural" a prévalu.

Au centre de la cour, une excavation quadrangulaire dont les parois et le fond sont recouverts d'une couche d'argile grise épaisse de $1 \mathrm{~m}$, destinée à l'étanchéifier, est interprétée comme un bassin dont les parois ont été récupérées. Les corps de bàtiment bordant la galerie à l'ouest, au sud et à l'est sont divisés en pièces rectangulaires de largeur variable. Ln sol de mortier blanc est conservé dans l'une des pièces du bâtiment ouest. La plus grande pièce de l'ensemble $(8,70 \mathrm{~m} \times 6,70 \mathrm{~m})$ est située immédiatement au nord, dans l'axe de la cour et du bassin. Elle est dotée d'un chauffage par hypocauste, alimenté depuis l'angle nord-est. L'affaissement partiel du lerrazzo, construit sur une fosse plus ancienne, a nécessité une réfection complète de l'installation.

La profondeur des fondations, supérieure à $1,70 \mathrm{~m}$ et parfois mème à $2 \mathrm{~m}$ - hormis celle de la paroi intérieure de la galerie - et l'épaisseur des couches d'éclats de taille liées à la construction permettent de penser que l'essentiel des élévations est désormais en pierre. L.implantation de cette vaste demeure a manifestement nécessité dimportants travaux daménagement et de nivellement du terrain : ainsi, les remblais d'installation du sol en mortier blanc reposent-ils directement sur le sable naturel. 


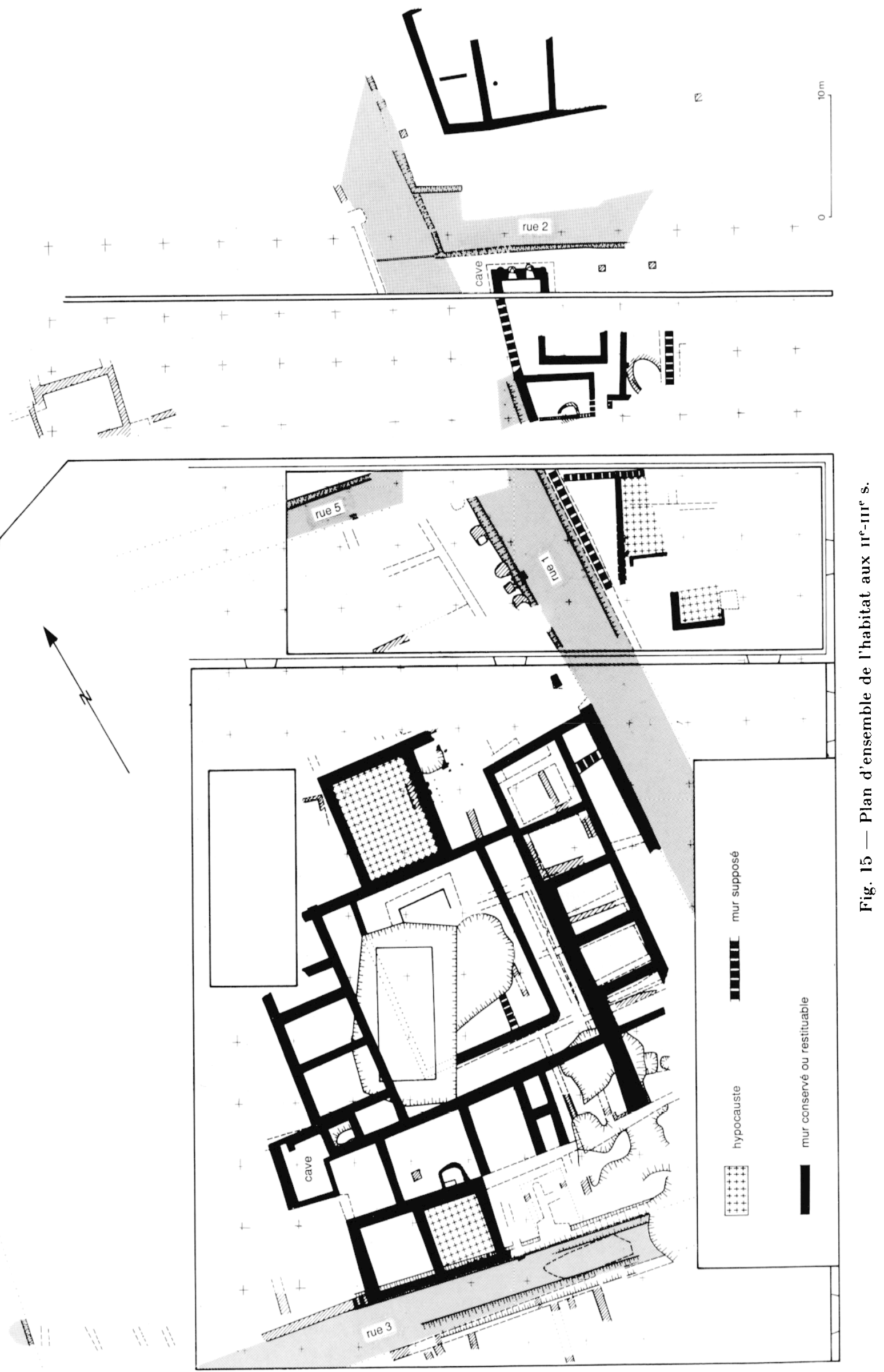




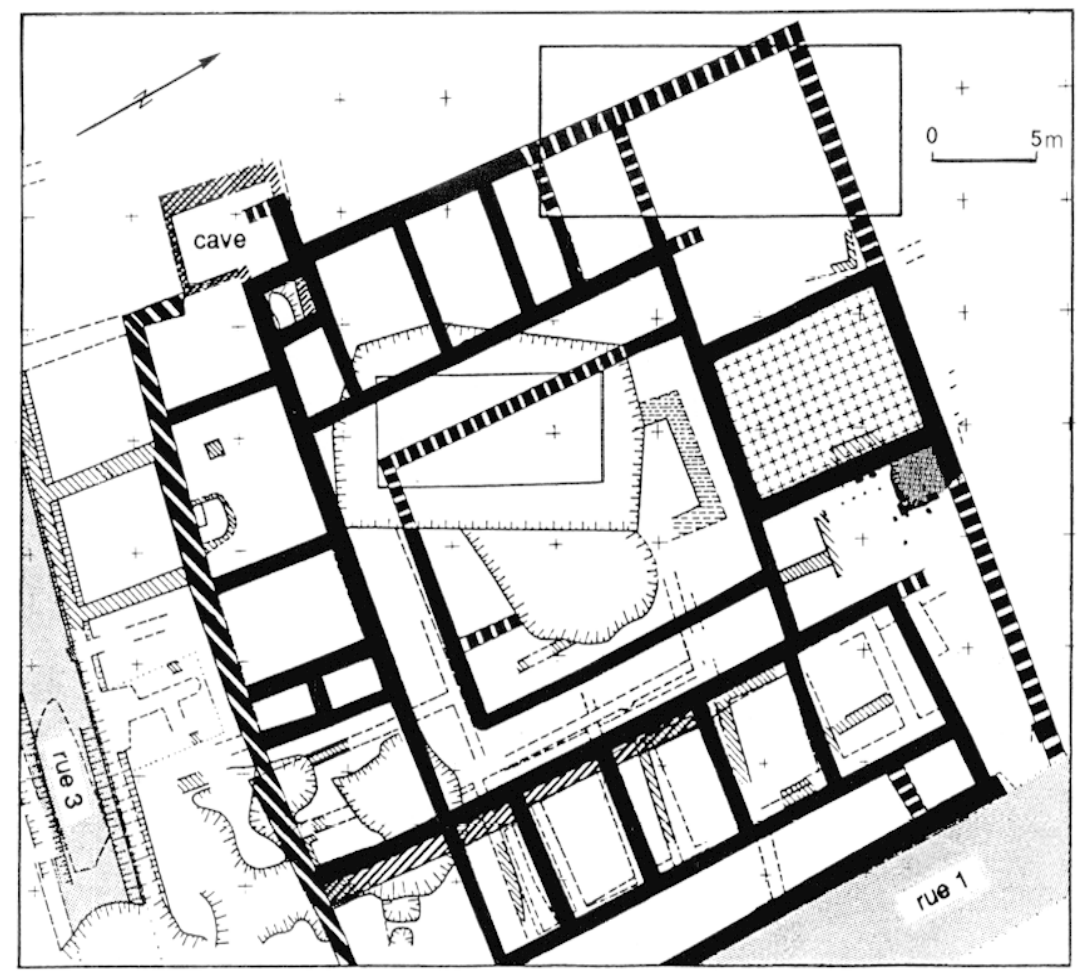

Fig. 16 - Domus de l'îlot B ( $\mathrm{II}^{\mathrm{e}} \mathrm{s}$ ) et ses remaniements postérieurs.

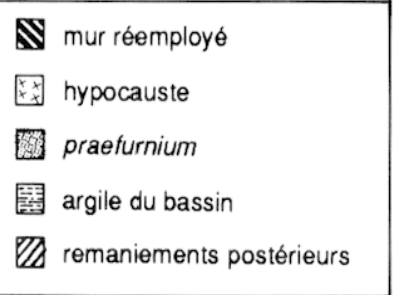

En raison de l'ampleur des récupérations et des arasements, c'est surtout l'observation de l'agencement des tranchées de récupération qui a conduit à reconstituer un plan dont la cohérence est la justification principale. La récupération des murs en une seule séquence et surtout la profondeur constante de leurs fondations, déjà mentionnée, confirment que ceux-ci appartiennent à un même ensemble. Il est cependant impossible d'apprécier les remaniements successifs. De même, si l'emprise de l'ancienne galerie-portique le long de la rue 1 apparaît effectivement intégrée à l'ensemble, son système de couverture tout comme l'existence éventuelle d'un etage restent incertains.

La disparition presque totale des niveaux d'occupation ne simplifie pas la datation de la domus. Toutefois, une fosse recelant du mobilier datable des années 60-70 à 110-120 est directement associée à sa construction, et scellée par le sol de mortier blanc. De plus, la réalisation du premier hypocauste au nord ne peut être placée avant la fin du $\mathrm{r}^{\mathrm{er}}$ s., et la destruction de son second état n'intervient pas avant la fin du $I^{\mathrm{e}} \mathrm{s}$. L'édification de ce vaste ensemble n'est donc pas antérieure à la fin du $\mathrm{I}^{\mathrm{er}} \mathrm{s}$. et son occupation se poursuit au moins durant tout le $\mathrm{II}^{\mathrm{e}} \mathrm{s}$.

Lne cave, dont seule la dernière occupation a pu ètre datée (fin $\mathrm{I}^{\circ}$ - milieu $\mathrm{II}^{\circ} \mathrm{s}$.), est établie dans un second temps à l'angle sud-ouest de la domus. Son accès, constitué - aux derniers temps de son utilisa- tion en tout cas - d'un simple plan incliné, nécessite le percement du mur ouest du corps de bâtiment occidental, mais cette observation n'implique pas forcément l'abandon du bâtiment. Les parois de cette cave rectangulaire ( $5 \times 3,40 \mathrm{~m}$ environ) sont constituées d'un seul parement de petit appareil, sans fondation. La destruction de la cave est visiblement consécutive à un incendie qu'attestent des traces de rubéfaction sur les murs, ainsi que l'importante couche de charbons de bois couvrant le sol, interprétée comme l'effondrement des poutres du plafond. Sous celle-ci, un matériel céramique relativement abondant a été découvert, écrasé en place sur le sol de mortier et de cailloutis (fig. 17, 3029). Il comprend notamment une amphore du type 50 de la classification de Peacock (Peacock, Williams, 1986), que l'on rencontre dans des contextes datant des années $250-350^{18}$. Un pied de table en pierre était disposé dans une fosse cuvelée carrée de $0,80 \mathrm{~m}$ de côté et de $0,30 \mathrm{~m}$ de profondeur (Burnand, 1984, p. 343 , fig. 5). De nombreux fragments de peinture murale provenant du remblai supérieur de la cave (3001) autorisent la restitution de deux parois (fig. 18); leur rubéfaction partielle manifeste leur probable appartenance au mème ensemble que la cave. Des comparaisons peuvent être établies avec

18 Identification de $\mathbf{M}^{\text {me }}$.J. Baudoux, à qui nous adressons tous nos remerciements. 


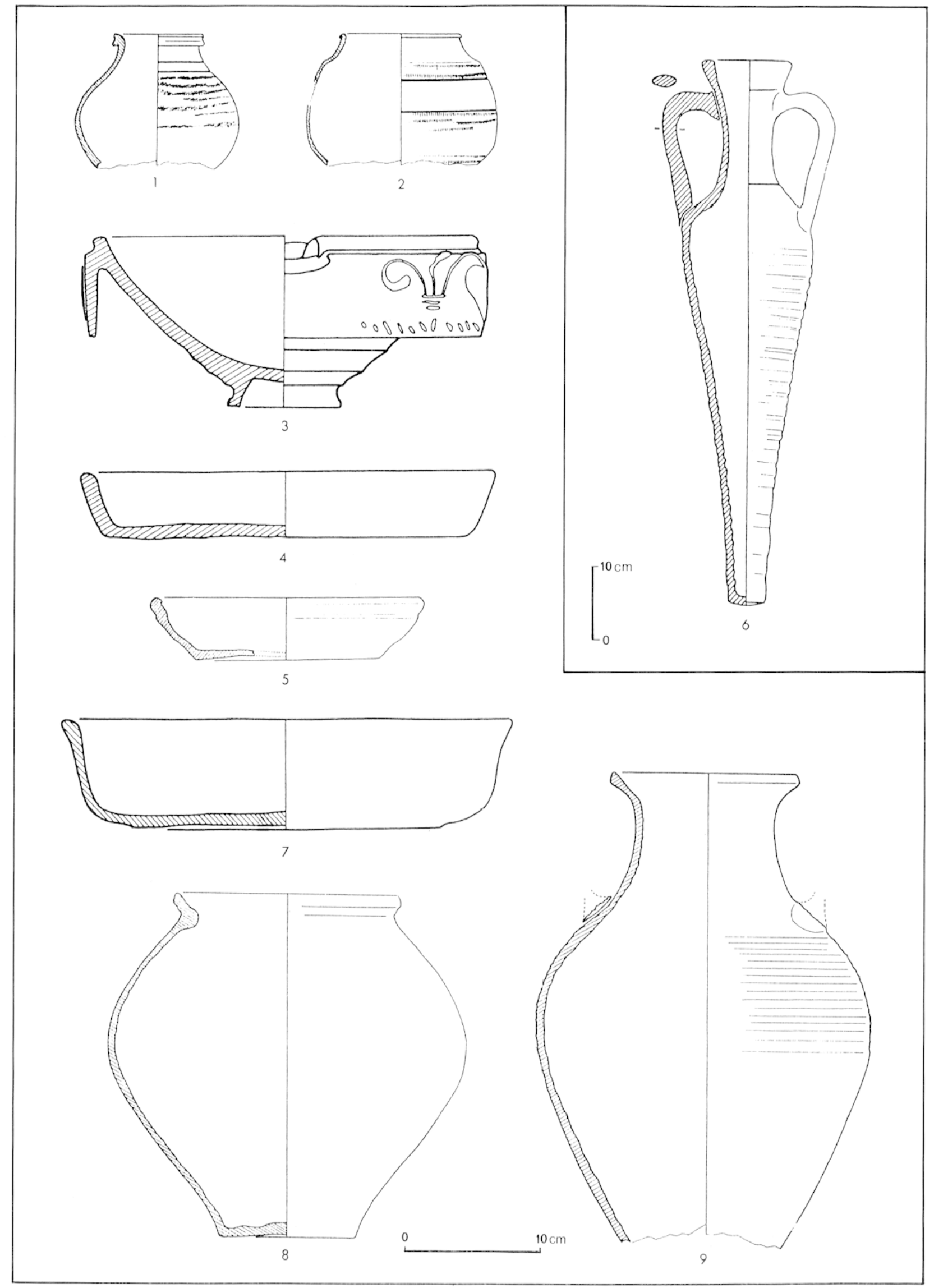

Fig. 17 - Céramique provenant de la cave située au sud-ouest de la domus (fin I"-milieu du II" s.). 3029 : 1. pàte claire, lissage partiel : 2, pàte rose, engobe brun légerement métallescent: 3. Drag. 43, sigillée; 4. commune rugueuse grise : 5 , commune rugueuse à degraissant siliceux, grise ; 6. amphore Peacock 50 . $3001: 7,8$, commune rugueuse à dégraissant siliceux, blanche: 9 , commune claire. 

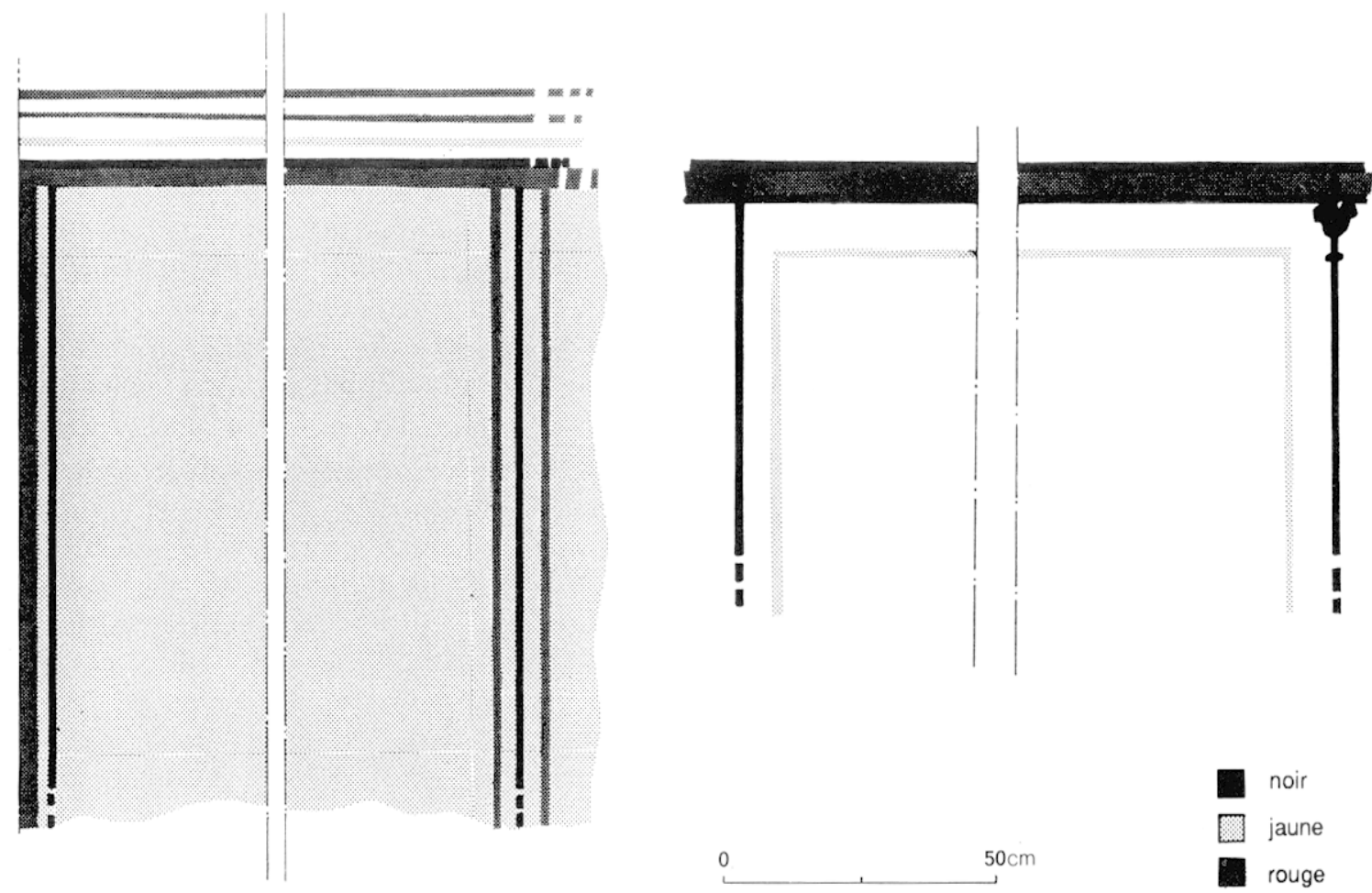

Fig. 18 - Restitution graphique de la peinture murale issue du comblement de la cave (3001).

deux décors. liun provenant du cryptoportique de Meikirch en suisse (I)rack, 1986, p. 46), l'autre de la villa du Grosswald à Sarreinsming en Moselle (Heckenbenner, 1983, p. 171), tous deux datés de la seconde moitié du II"s.

Ln autre élément témoigne peut-ètre d'un remaniement tardif de la domus; il s'agit d'un mur qui vient doubler à l'est la paroi intérieure du corps de bàtiment oriental. Suivi sur une vingtaine de mètres, ce mur moins profondément fondé est presque totalement récupéré; aucun indice ne permet d'en préciser la datation.

Le plan de la résidence de l'Arsenal est visiblement conforme à des modèles méditerranéens; la cour intérieure avec galerie, dont nous avons déjà souligné la forme géométrique parfaite, et le bassin evoquent le peristyle de certaines domus romaines. La vaste salle chauffée située dans l'axe est vraisemblablement une pièce d’apparat préservée des rigueurs hivernales et profitant de la fraicheur de l'eau en été. Cette disposition centrée et axée apparait fréquente parmi les exemples de comparaison recensés en Gaule du centre ou du nord, depuis un prototype comme la grande maison de Bibracte datée du $\mathrm{I}^{\mathrm{er}}$ s. avant J.-C. (Goudineau, Kruta, 1980, p. 226), jusqu à des demeures de datation proche de celle de l'Arsenal. comme par exemple :
- à Cologne, la maison à la mosaïque de Dionysos, de plan plus trapézoïdal (Fremersdorf, 1956: Precht, 1971) et la maison à atrium, de surface comparable (Precht, 1971, p. 5̄5-56);

- à Lyon, rue des Farges, la maison aux masques dont la très grande salle de réception est légèrement désaxée (Desbat, 1984, p. 45-49).

Cne maison de plan-masse très comparable, à Caerwent (Grande-Bretagne), possède de même que ('elle de l'Arsenal une galerie fermée accolée au corps de bàtiment oriental et domnant sur la rue, interprétée comme un "portique d'entrée" (Wacher, 1974. p. $384-38 \%)$.

A Metz, rue Marchant, une cour intérieure pourvue d'un bassin proche de celui de l'Arsenal est datée du dernier tiers du $\mathrm{I}^{\mathrm{er}} \mathrm{s}$. (Brunella el alii, 1988 , p. 36) : cette découverte rend probable l'existence à Metz d'autres résidences du même type.

La conception d'ensemble, inspirée de modèles romains ou méridionaux, l'ampleur des travaux de terrassement et de maçonnerie et l'importance de la surface construite sont autant d'indices du rang social du propriétaire de la domus de l'Arsenal. Celle-ci jouxte des constructions de surface beaucoup plus modeste (habitats, boutiques?) : à Metz comme ailleurs, les grandes demeures urbaines ne semblent 


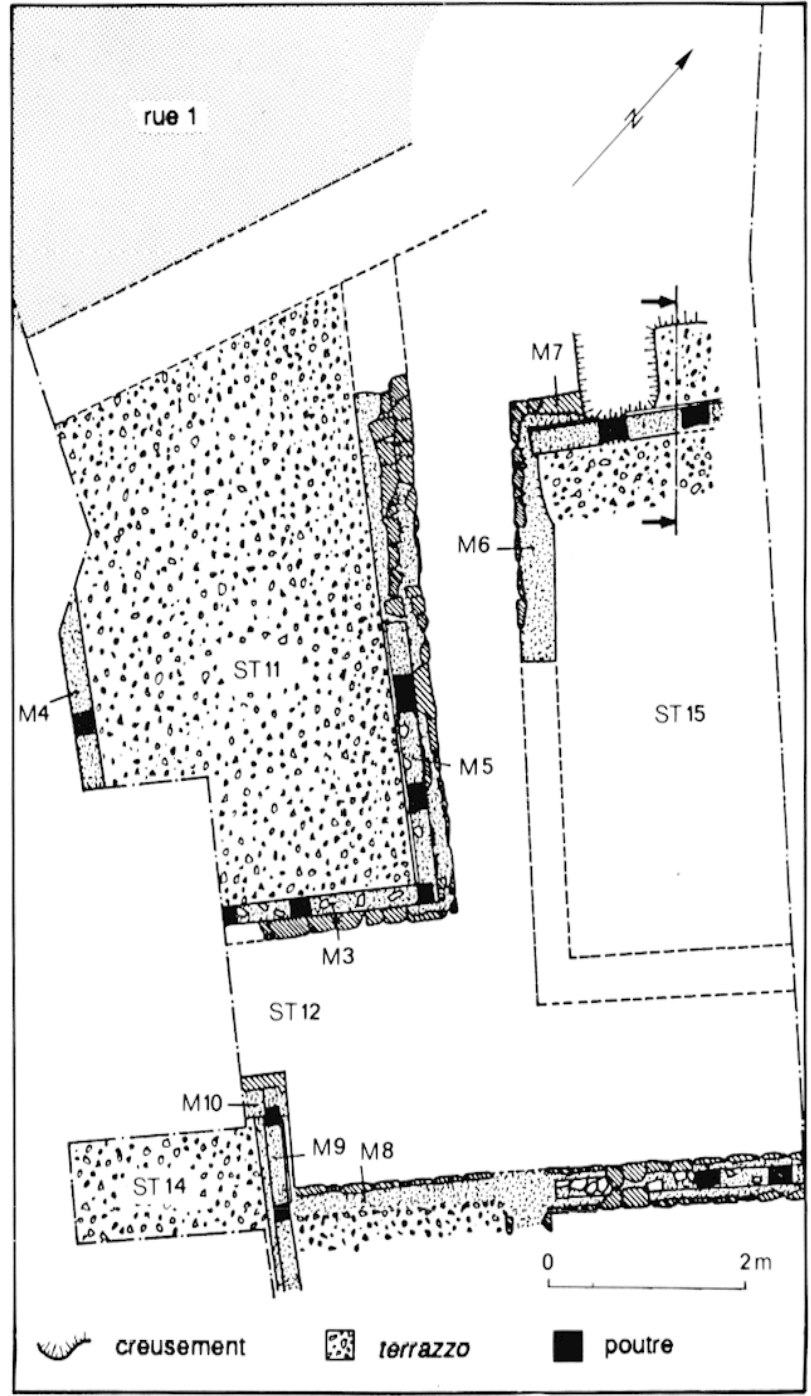

Fig. 19 - Habitat nord de l'îlot D (fouille 1986).

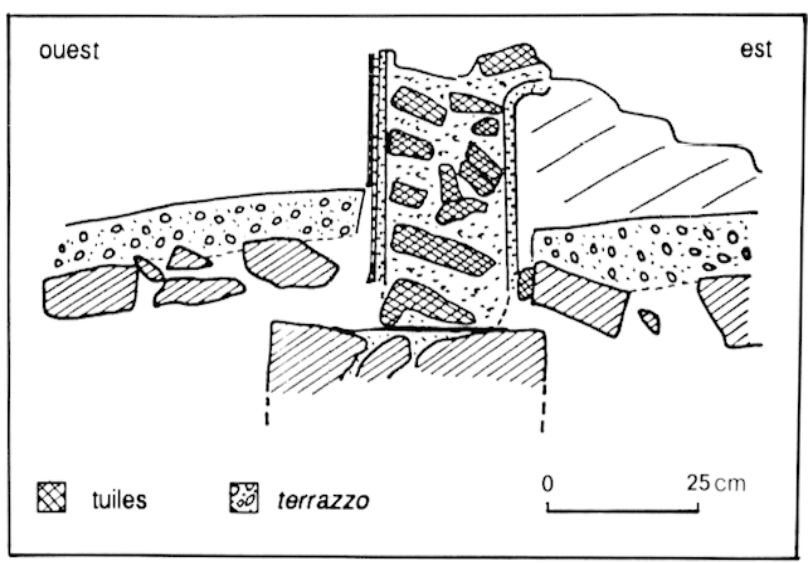

Fig. 20 - Section du mur M7. pas se regrouper en des quartiers socialement privilégiés (Goudineau, 1980, p. 300).

La rive sud de l'îlot $B$ demeure mal connue; seul un ensemble de deux pièces, sur lequel s'appuie l'angle sud-ouest de la domus, apparaît cohérent. Les radiers de fondation des murs, en calcaire bleu du sinémurien, sont d'une largeur moyenne de 0,70 à $1 \dot{\mathrm{m}}$. Le sol a disparu dans la pièce ouest; à l'est, un terrazzo d'hypocauste, reposant sur un hérisson de pierres bleues, est implanté directement sur le terrain géologique. Attenante au nord à cette seconde pièce, une structure quadrangulaire dont le sol de béton porte les traces d'arrachage d'un foyer est interprétée comme un praefurnium. Les vestiges d'un second conduit de chaleur, apparemment contemporain du premier, sont visibles dans le terrazzo à l'est (briques plates disposées de chant). L'installation de chauffage a dû être rénovée ou supprimée lors de l'élablissement de la domus, nécessitant la destruction du praefurnium nord.

\section{L'îLOT D}

La fouille a, semble-t-il, porté sur deux unités d'habitation mitoyennes mais les fondations de l'Arsenal n'ont pas permis de vérifier ce point. L'habitat sud, très fortement recoupé par des structures postmédiévales, n'a pas fait l'objet d'une étude approfondie; il comportait un hypocauste mesurant $6,50 \mathrm{~m}$ de côté sur l'axe nord-sud. L'habitat nord, mieux conservé, pourrait occuper une parcelle trapézoïdale d'environ $10 \times 17 \times 15 \times 17 \mathrm{~m}$. Toutes les cloisons conservées ont une élévation en matériaux légers sur sablière basse, reposant sur des solins maçonnés (fig. 19).

Ces derniers sont construits en pierres calcaires bleues liées au mortier. D'une profondeur de $1 \mathrm{~m}$ (5 à 8 assises) et parfaitement horizontaux (un seul d'entre eux présente un dénivelé de $4 \mathrm{~cm}$ entre ses deux extrémités sur une longueur de $5 \mathrm{~m}$ ), ils sont parementés sur les deux côtés. La sablière a laissé une fine couche brune et grasse visible entre le solin et l'élévation des murs M5, M7 et M8. Sa décomposition a entraîné un affaissement d'une quinzaine de centimètres de l'élévation, comme le montre la coupe du mur M7 (fig. 20) : le point de jonction légèrement débordant du premier enduit peint côté ouest avec le sol contemporain se retrouve $14 \mathrm{~cm}$ en dessous de ce sol, alors que l'enduit peint du second état, associé à un sol disparu, se positionne $1 \mathrm{~cm}$ seulement au-dessus du premier sol.

L'ossature de l'élévation est constituée de poutres verticales fichées dans la sablière, retrouvées à l'état de charbon de bois. Leur écartement est généralement constant (deux fois $0,54 \mathrm{~m}, 0,50 \mathrm{~m}$, 
$0,60 \mathrm{~m})$. Celles de M9 sont plus éloignées l'une de l'autre $(0,74 \mathrm{~m})$; le poteau d'angle des murs 3 et 5 est espacé de $1,04 \mathrm{~m}$ de la première poutre de M3 et de $0.68 \mathrm{~m}$ du poteau suivant de $\mathrm{M5}$. De forme carrée, elles sont de dimensions très proches (en moyenne $22 \mathrm{~cm}$ de côté); trois d'entre elles ont un gabarit supérieur $(30 \times 18,26 \times 20$ et $24 \times 18 \mathrm{~cm})$.

Le bourrage entre les poutres verticales est constitué de fragments de tuiles (tegulae et imbrices) disposés en assises horizontales liées par un mortier de chaux et de terre limoneuse. Il est possible, d'après la nature des remblais de destruction, qu'à partir d'une certaine hauteur, cette maçonnerie sommaire ait été remplacée par un mur en terre ${ }^{19}$. Ces élévations sont larges de 20 à $24 \mathrm{~cm}$ en moyenne, M10 atteignant $27 \mathrm{~cm}$ d'épaisseur.

Pour la plupart, les parois sont revêtues d'enduits peints. Le couloir St 12 qui distribue les différentes pièces possède dans un premier temps des plinthes jaunes mouchetées (M3, M5 et M8) et des panneaux blancs (M7 et M9) à décor géométrique noir et rouge $(M 7)$. Dans un second état, trois parois montrent des traces de réfection : les murs M3 et M7 sont recouverts d'un enduit brossé blanc et M9 est repeint en rouge.

La structure 11 présente d'abord une plinthe jaune mouchetée très dégradée, revêtue d'un enduit blanc; le parement sud de M4 est peint en rouge. La structure 14 conserve une peinture de bonne qualité où alternent d'étroits panneaux rouges et de larges panneaux noirs à décors de thyrses croisés.

Les sols sont constitués d'un agrégat de graviers, petits fragments de tuiles et mortier; ils reposent sur un hérisson de pierres calcaires bleues recouvertes de sable et de fragments d'enduits peints. Ce radier a été construit dès l'achèvement des murs alors que la couche supérieure du sol a été mise en place après l'exécution des enduits peints.

L.es pièces étaient pratiquement vierges de tout élément associé à l'occupation; nos principaux arguments de datation sont fournis par l'analyse stylistique des peintures, et notamment du décor de thyrses croisés. Bien que celui-ci apparaisse en Italie et dans le sud de la Gaule dès la première moitié du $\mathrm{I}^{\mathrm{er}} \mathrm{s}$. (Barbet, 1982, p. 59-62), il ne semble s'imposer dans les provinces septentrionales de l'Empire qu'à

19 A Aventicum, pour une cloison de refend en colombage, de technique tout a fait comparable, la nature des matériaux de démolition rencontrés indique au contraire la présence d'un hourdis maçonné sur toute la hauteur de son élévation (Morel, 1988, p. 51, fig. 29). partir de la fin du $\mathrm{I}^{\mathrm{er}}$ ou de la première moitié du $I^{\circ}$ s. Les plinthes du cryptoportique de Buchs en Suisse datées des années 100-140 (Drack, 1986, p. 3940) et celles du temple II de Elst construit après 70 (Bogaers, 1955, pl. 22) présentent des analogies certaines. Mais c'est surtout dans le secteur central de la villa de Saint-Lilrich (Moselle) qu'il faut chercher les comparaisons les plus pertinentes. Ces peintures, encore inédites, ont été exécutées dans la première moitié du II $^{\circ}$ s. Au-dessus de plinthes jaunes mouchetées, des panneaux rouges alternent avec des panneaux noirs à décor de thyrses croisés. Ces quelques parallèles permettent de proposer une datation dans la première moitié du $\mathrm{II}^{\mathrm{e}} \mathrm{s}$.

Une cave construite en petits moellons de calcaire blanc a été partiellement fouillée à l'angle de la parcelle (fig. 15). Son étanchéité est renforcée par le placage contre la paroi externe d'une couche d'argile épaisse de $0,50 \mathrm{~m}$ environ. Le mur nord comporte deux soupiraux formés chacun de deux blocs de calcaire jaune taillés; des traces de barreaux sont observables sur ces blocs. Dans cette cave était enfoui un grand pot ovoïde en céramique commune dont l'ouverture était soigneusement obturée par une tuile plate à fleur de sol. Le comblement de la cave, formé de débris de maçonnerie ainsi que de tuiles, parfois entières, amoncelées au pied des soupiraux, est datable du ${ }_{11 I^{*}} \mathrm{~s}$., mais les relations avec l'habitat précédemment évoqué ne sont pas connues.

Ln artisanat métallurgique daté de la seconde moitié du $1 \mathrm{I}^{\mathrm{F}} \mathrm{s}$. succède à la destruction de l'habitat, dont les murs ouest et est semblent encore conservés en élévation. Deux horizons successifs ont été distingués. Le plus ancien est matérialisé par de nombreux foyers allongés (foyers de forge?) et deux foyers circulaires (vestiges de bas fourneaux ?). Le second, qui a livré une quinzaine de monnaies en bronze des années $260-274^{20}$, se caractérise par sa richesse en battitures, qui traduit une activité de forge au sens strict du terme; plusieurs structures excavées ou construites lui sont associées, ainsi qu'un bloc de calcaire quadrangulaire de $0,60 \times 0,60 \times 0,30 \mathrm{~m}$ (support d'enclume?). A chacun de ces horizons, correspond un caniveau, qui amenait vraisemblablement l'eau nécessaire aux différents secteurs de l'atelier. L'analyse des déchets prélevés montre l'exis-

20 Nous remercions vivement M. M. Amandry, conservateur au Cabinet des Médailles, qui a bien voulu assurer l'identification de l'ensemble des monnaies de la fouille de l'Arsenal. 
tence d'une chaîne sidérurgique complète, de la réduction du minerai de fer au travail du métal; le travail du bronze est également attesté. L'ensemble de ces résultats a fait l'objet d'une publication spécifique (Leroy et alii, 1990). Sur le plan de la topographie urbaine, il nous apparaît interessant de souligner l'existence d'une métallurgie de réduction en plein cœur d'un espace urbain. Il ne s'agit certes pas là d'un fait isolé ; cette installation implique cependant un approvisionnement régulier en minerai et combustible, l'évacuation de la majorité des déchets et un certain nombre de nuisances qui niont apparemment pas constitué un obstacle insurmontable.

\section{L'îLOT E}

Au carrefour des rues 1 et 2 , une unité d'habitation d'une dizaine de mètres de large a été partiellement étudiée. Donnant sur la rue 1 , la pièce la plus occidentale peut ètre considérée comme un sous-sol, du fait de son encaissement. Dans un second état,

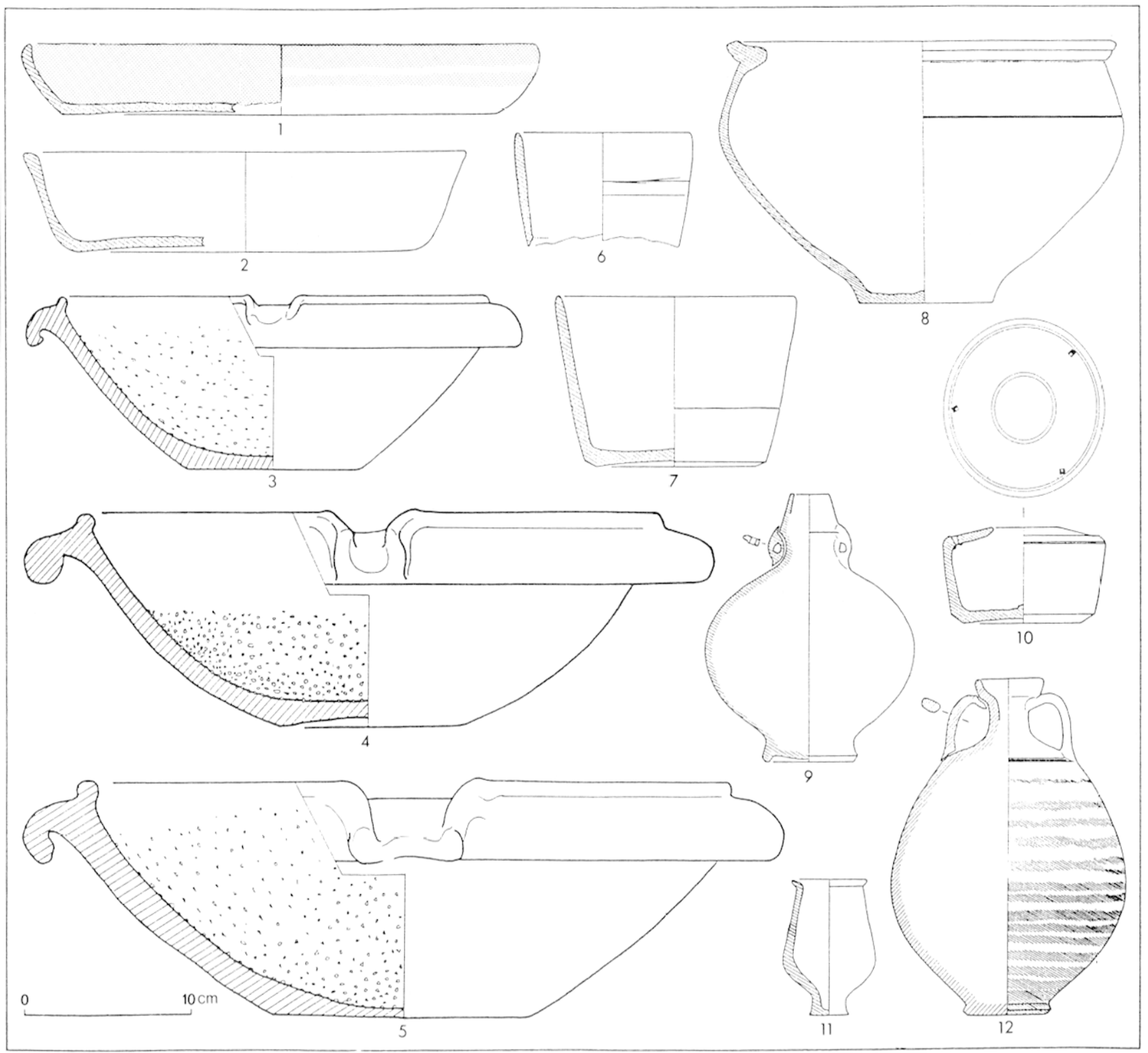

Fig. 21 - Ensemble de céramique provenant de la pièce située à l'angle ouest de l'îlot $\mathrm{E}$ (III"s.) : 1. pàte rose, engobe rougeatre: 2 , commune rugueuse à dégraissant siliceux, grise ; $3-5$, mortiers en pàte rose; 6. 7. sigillée de Caule de l'Est : 8 . commune rugueuse à dégraissant siliceux, blanche: 9 . Ludowici type Kb, sigillée: 10. sigillèe de Ciaule de l'Fst: 11, commune a pate rose, engobe rose ; 12, commune grise, lissage partiel. 
seul le tiers sud, limité par une cloison de planches, est utilisé alors que le reste de la pièce est comblé d'un remblai riche en enduits peints. Son abandon final semble consécutif à un incendie. qui est attesté par une couche de destruction composée de charbons de bois sur $0,15 \mathrm{~m}$ d’épaisseur. Celle-ci contenait un abondant mobilier céramique, attribuable au ${ } \mathrm{I}^{\mathrm{e}} \mathrm{s}$. (fig. 21).

La pièce suivante à l'est, dont le sol se situe $0.55 \mathrm{~m}$ plus haut, est de plain-pied avec le trottoir, en raison de la pente naturelle. Elle comporte un foyer constitué de tuiles plates liées à l'argile, et son occupation peut ètre datée de la fin du $11^{\mathrm{e}} \mathrm{s}$.

L implantation de caves médiévales a totalement oblitéré les vestiges antiques situés plus à l'est.

\section{TOPOGRAPHIE URBAINE : UN QUARTIER AU SCHÉMA ORIGINAL}

La découverte de quatre rues, reconnues sur une longueur significative, et d'un fossé d'une cinquième renouvelle considérablement nos connaissances sur lorganisation du quartier.

Comme le pressentait R. Jolin (1977, p. 25 n. 8), le réseau, vraisemblablement orthonormé, du centreville ne se poursuit pas dans le secteur de l'Arsenal. I.a rue 1 diverge de plus de $27^{\circ}$ vers l'ouest, et les rues 3 et 5 , qui forment respectivement avec cette dernière des angles de $80.5^{\circ}$ et $81^{\circ}$, sécartent donc d'environ $16^{\circ}$ de la rue 2 et de l'axe supposé des decumani. Ce système ne semble pas généré par la voie de Scarponne: d'une part, le tracé proposé traditionnellement pour cette voie est d'orientation sensiblement différente: d'autre part, la voirie et les habitats a l'est de la rue 1 , c'est-à-dire compris entre cette dernière et la voie de Scarponne, respectent les orientations du centre de la ville.

La rue 2, dont l'existence etait envisagée par R. Jolin (1977, p. 24-26) s'intègre à la trame proposée pour le centre-ville actuel. Elle permet de connaitre avec une certaine approximation - due aux difficultés de recalage des relevés de 1964 l'entraxe de deux decumani (rue 2 et rue découverte en 1964 , fig. 4) : $115 \mathrm{~m}$, soit environ 388,5 p.m., si le $p . m$. a bien été la mesure utilisée lors de l'implantation du réseau. L,îlot compris entre ces deux decumani mesure donc environ $109 \mathrm{~m}$, ce qui correspond à 368.2 p.m. Cette dimension évoque 3 actus. soit 360 p.m., mais l'écart non négligeable $(2,28 \%)$ et limprécision relative de la mesure ne permettent pas de conclure : alors qu'à Aventicum, beaucoup d'insu- lae mesurent $3 \times 2$ actus $(106.5 \times 71 \mathrm{~m})$ (Frere, 1977 , p. 91). dautres modules de dimensions proches semblent être des multiples de 5 ou 10 pieds : $250 \times 370$ pieds à Ariminum, $270 \times 385$ à Bononia ... (Duncan-Jones, 1980, p. 129).

Les rues $1,3,4$ et jófinissent un îlot trapézoïdal, dont les còtés est et sud mesurent respectivement $62 \mathrm{~m}$ et $69 \mathrm{~m}$ environ. Ses dimensions, nettement inférieures à celles proposées pour les îlots du centre de la ville (Jolin, 1977, p. 24), sont supérieures à celles de lî̀lot partiellement reconnu au Pontiffroy : $43 \times 39 \mathrm{~m}$ (Waton, 1986, p. 90). Sa morphologie, imposée par l'articulation de deux réseaux d'orientation différente, ne permettant pas de préjuger de l’organisation générale du quartier, les données des fouilles anciennes et le cadastre ont été réexaminés, afin de déceler d'éventuels indices susceptibles de nous aider à restituer la topographie urbaine. Ces travaux se sont révélés fruclueux. Un alignement se dessine en effet sur près de $80 \mathrm{~m}$, que définissent la façade occidentale de Saint-Pierre-auxConnains, le parcellaire qui jouxte l'édifice au sud, le mur est du "couloir" du IV" s. dégagé par J.-J. Hatt (Hatt, 1962, p. 496), et la limite ouest du cloître médiéval (fig. 2\%). Il s'avère que cet alignement, manifestement d'origine antique, est le symétrique parfait de la limite est de l'îlot $B$ (galerie en façade comprise) par rapport à l'axe restitué de la rue 4 ( $\mathrm{si}$ on lui attribue arbitrairement une largeur de $6 \mathrm{~m}$ ). De plus, l'extrémité sud de cet alignement est marquée par un retour approximativement perpendiculaire, qui rejoint la prolongation de la façade sud de l'îlot. B. Ces données convergentes nous ont conduits à envisager l'hypothèse d un axe, vraisemblablement de voirie, symétrique de la rue 1 par rapport à la rue 4, abandonné dès l'Antiquité tardive. mais dont le tracé se serait maintenu dans le parcellaire jusqu'à nos jours.

Quelques points peuvent conforter cette hypothèse. Ainsi, J.-.J. Hatt signale-t-il la présence de "gros blocs calcaires taillés", sur lesquels reposent les fondations de Saint-Pierre-aux-Nonnains et qui correspondent peut-être aux blocs de sa figure 15 , bordant à l'ouest la façade de l'édifice (Hatt, 1960, p. 217-219). I'autre part, trois blocs quadrangulaires, régulièrement espacés de $2,60 \mathrm{~m}$ environ, semblent, d'après les relevés, disposés sur un mur de "soutènement", daté du Haut-Empire et parallèle à la façade de Saint-Pierre-aux-Nonnains (Hatt, 1962, fig. 24). Ce mème mur de "soutènement" est parfaitement aligné avec le mur ouest de l'ensemble du IV"s., distant de près de $25 \mathrm{~m}$, ce qui manifesterait à nouveau la reprise durant l'Antiquité tardive d'un 


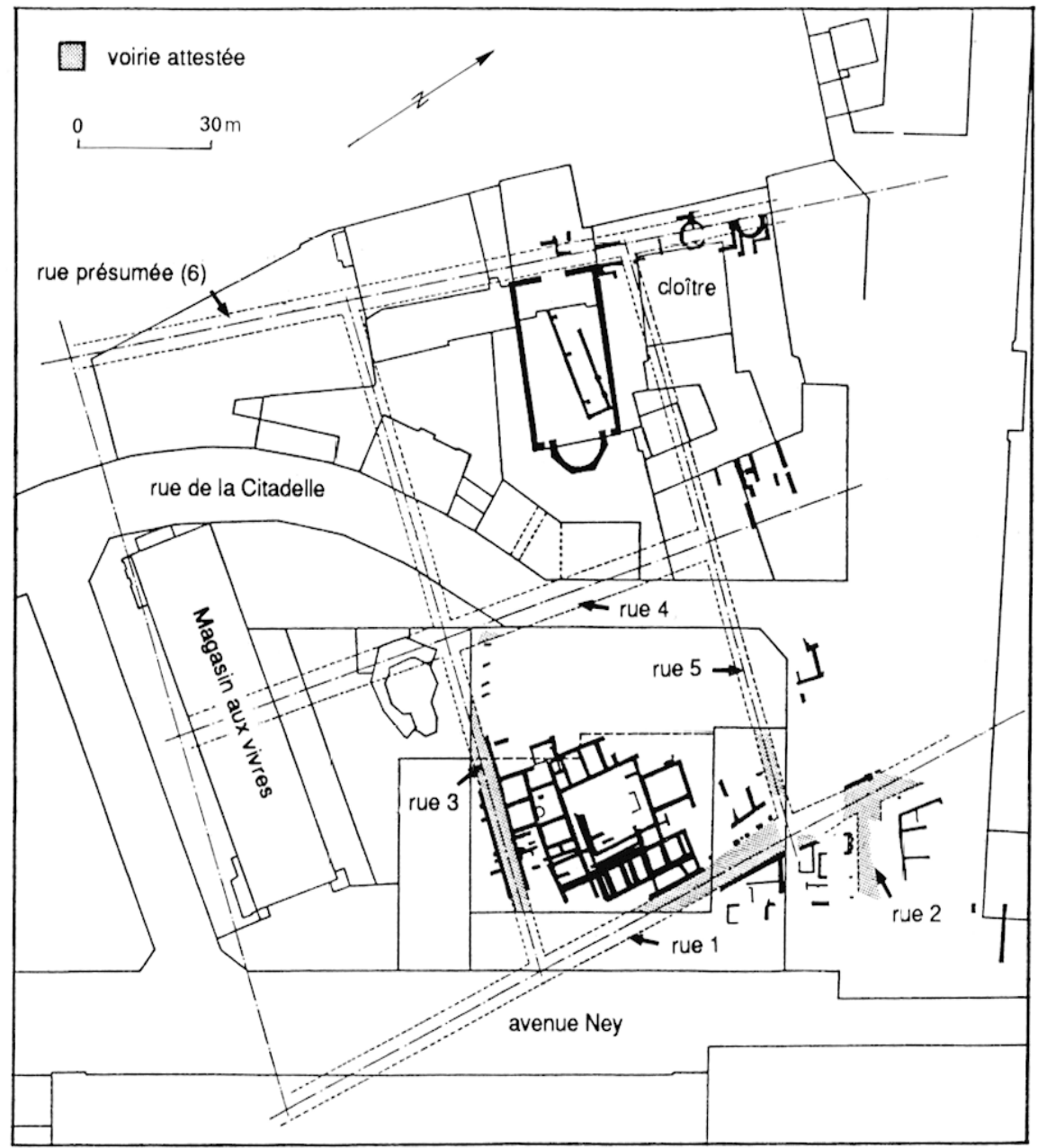

Fig. 22 --

Proposition de restitution de la voirie du quartier. axe antérieur. L'intervalle entre ces deux alignements, de l'ordre de $5,50 \mathrm{~m}$, est identique à la largeur - caniveaux compris - de la rue 1 . Les dés de pierre pourraient ainsi appartenir à d'anciens portiques, bordant la chaussée supposée.

Le report du tracé des rues $1,2,3,4,5$ et de la rue présumée 6 sur le plan topographique de la ville montre l'étroite adéquation de certains de ces tracés et du relief : l'axe 6 longe la rupture de pente prononcée qui domine la Moselle, et la rue 4 borde l'autre flanc de la butte (fig. 23) ${ }^{21}$. Cependant, ce facteur ne suffit pas à expliquer l'organisation d'ensemble de la voirie du quartier. La rue 1 , en effet, ne suit pas les courbes de niveau et ne constitue pas non plus un axe médian entre la rue 4 et la voie de Scar-

21 Rappelons que le dénivelé brutal au nord est dû à l'aménagement de l'Esplanade au début du $\mathrm{XIX}{ }^{\mathrm{e}} \mathrm{s}$.; la pente était plus régulière durant l'Antiquité, comme l'ont montré les travaux de G. Collot sur le chantier de l'École des arts appliqués (Collot, 1967-1968, p. 8). ponne, ce qui aurait permis de réduire l'aspect trapézoïdal des îlots. Son tracé est peut-être imposé par la nécessité de desservir - ou de contourner - un équipement important (monument public?) dont la nature et l'emplacement précis nous échappent.

L'édification de l'enceinte du Bas-Empire, une trentaine de mètres à l'ouest, peut expliquer la désaffection de la rue 6 supposée. Tout aussi plausible demeure l'hypothèse d'une restructuration du secteur imposée par un projet urbanistique indépendant d'impératif́s défensifs : ensemble thermal du IV $V^{\prime}$ s., voire "nymphée" antérieur d'identification délicate en raison de son très mauvais état de conservation (Hatt, 1961, p. 15). A Trèves, les fouilles menées par W. Reusch dans l'Aula Palatina ont montré que l'abandon des deux rues du HautEmpire qui se croisaient à l'emplacement du monument remonterait sans doute au II" $^{\prime \prime}$., lors de la construction d'un premier groupe de bâtiments publics (Reusch, 1955, p. 194-196). A Metz, l'espace réorganisé voit s'implanter à partir de 370 ( \pm 15 ans) le vaste ensemble thermal auquel semble se rattacher 
Fig. $23-$ Topographie des quartiers sud de la ville

(d'après un document du Centre national d'archéologie urbaine).

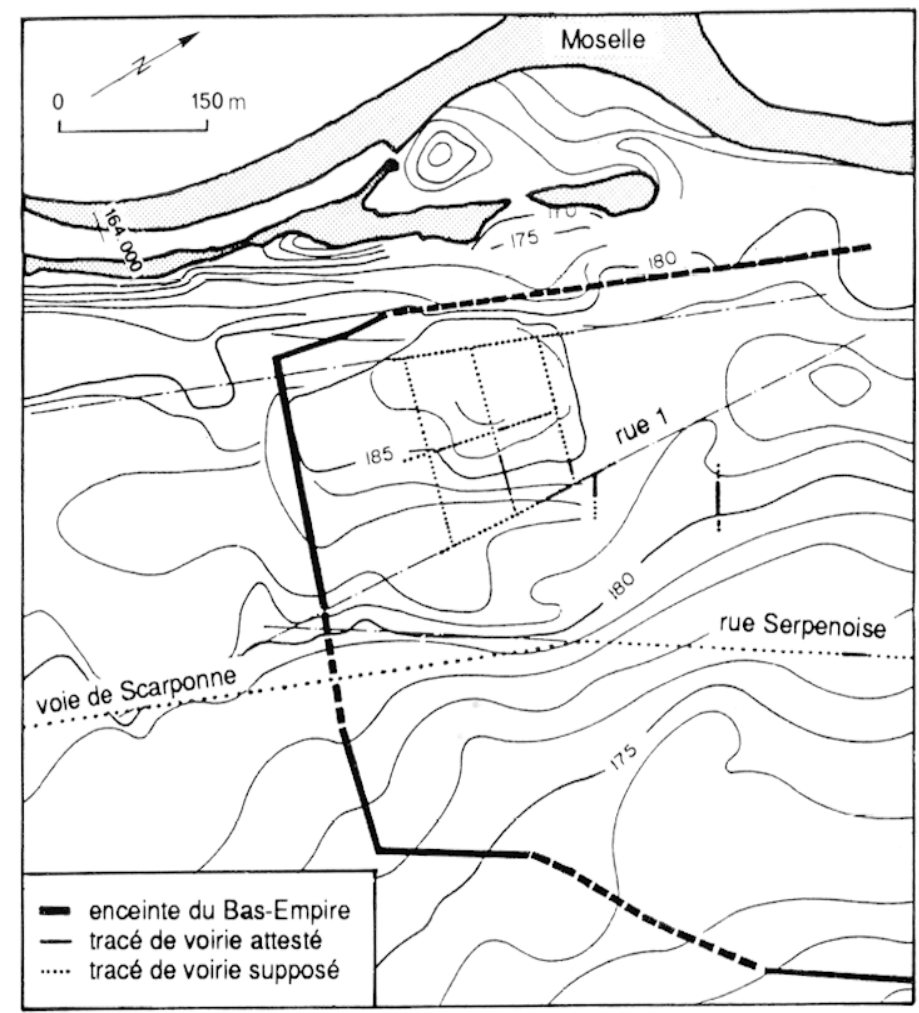

le bâtiment à plan basilical de 30 ans postérieur, qui deviendra Saint-Pierre-aux-Nonnains (Delestre, 1988a, p. 20-28).

L'installation de deux latrines post-médiévales le long de la rue 3 , dont elles respectent le tracé, peut indiquer la perduration de cet axe, au moins jusqu'au milieu du $x^{\prime}{ }^{e}$ s. L'orientation du Magasin aux Vivres, édifié en 1564 , est également très proche de celle des rues 3 et 5 . Pérennise-t-elle un tracé antique, qui formerait avec les rues 3 et 5 un réseau régulier de voiries d'orientation ouest-nord-ouest/ est-sud-est, parallèles et équidistantes?

A l'issue des campagnes de fouille menées de 1983 à 1987, le quartier de l'Arsenal apparaît désormais comme l'un des deux secteurs les mieux documentés de la ville antique, avec le Pontiffroy (Waton, 1986). La mise au jour sur une longueur significative de quatre rues, le dégagement ponctuel d'un cinquième élément de voirie et le réexamen des données de fouilles anciennes autorisent une restitution provisoire de l'organisation de la moitié nord du quartier. Le système envisagé, qui remonterait à la première moitié du $\mathrm{I}^{\mathrm{er}} \mathrm{s}$., semble en partie lié à la topographie assez accidentée du site; il reflète sans doute également des objectifs plus proprement urbanistiques, que notre connaissance lacunaire de l'orga- nisation générale de la ville nous empêche de préciser.

L'ouverture de $4000 \mathrm{~m}^{2}$ d'un seul tenant a permis pour la première fois à Metz d'appréhender le parcellaire et son évolution sur près du tiers d'un îlot urbain; l'ampleur des récupérations a cependant considérablement limité nos observations dans le domaine des techniques de construction. Ce quartier ne semble pas réservé au Haut-Empire à l'habitat et à l'artisanat. Le "nymphée», d'interprétation discutable, et surtout les deux hypocaustes de plus de $11 \mathrm{~m} \times 20 \mathrm{~m}$ et d'au moins $25 \mathrm{~m} \times 10 \mathrm{~m}$, découverts lors des fouilles de l'École des arts appliqués (Collot, 1967-1968, p. 6, 18) témoignent de l'exis-

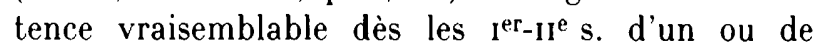
plusieurs ensembles publics.

L'érection dans la seconde moitié du Ive $s$. d'un ensemble monumental (thermes) manifeste l'abandon partiel du réseau de voirie du Haut-Empire - si notre hypothèse est juste. L'ampleur et la qualité des travaux alors engagés témoignent de la vitalité de la cité à la fin du Bas-Empire, vitalité que les fouilles récentes ne peuvent confirmer mais qui explique, tout autant que l'édification de l'enceinte, les profonds remaniements que connaît alors le quartier.

D. H., Ph.B., M.L., M.M., P.T., S. B., J.-M. B., I. B., O. F., M.G.-L., G. G., V. K., Cl.P., Gl. V. 


\section{BIBLIOGRAPHIE}

Barbet A.

1982 : La diffusion du IIJ" style pompéien en Gaule. Ire partie. Gallia, 40, p. 52-82.

Bayard D., Massy J.-L.

1983: Amiens romain, Samarobriva Ambianorum. Revue Archéologique de Picardie. $n^{\circ}$ spécial. $374 \mathrm{p}$.

Bogaers J. E. A.

1955 : De gallo-romeinse tempels te Elst in de Over-Betuwe, La Haye, Nederlandse Oudheden, 1.

\section{Bourger I., Cabart $\mathrm{H}$.}

1990 : La céramique et le verre de deux ensembles clos des XIV" et XvI" siècles à Metz (Moselle), Revue Archéologique de l'Est et du Centre-Est. 41, 1, p. 105-140.

Brunella Ph. et alii

1988 : Melz. Cinq années de recherches archéologiques, 1982-1987. Met\%, Éd. Iirection des Antiquités Ilistoriques et Préhistoriques de Lorraine, Groupe ('niversitaire. Vessin de Recherche Archéologique. 80 p.. 81 fig.

\section{Burnand Y.}

1984: Informations archéologiques. (Circonscription de Lorraine, Gallia, 42, p. 339-344.

Carré F., Gerber F.

1990 : Évreux (Eure) - Rue Saint-Louis, in : De la (iaule à la Vormandie, 2000 ans d'llistoire. 30 ans d'archéologic, catalogue d'exposition, Rouen, Éd. Musées et Monuments départementaux de la Seine-Maritime, p. 95-97.

Collot G.

1964 : Fouilles archéologiques sur le chantier du parking souterrain de l'Esplanade et rue Poncelet, Annuaire de la Sociëlé d'IIistoire et d'Archéologie de la Lorraine. I.XIV. p. 41-76.

1967-1968 : Fouilles archéologiques sur le chantier de l'École des Arts Appliqués, Annuaire de la Socièté d'Histoire el d'Archéologie de la Lorraine. L.XVII-I.XVIII, p. 5-32.

\section{Delestre X.}

1988a : Saint-Pierre-aux-Vonnains, Paris, Éd. Ministère de la Culture et de la Communication, Imprimerie Nationale, Guides Archéologiques de la France, 15, 64 p.

$1988 \mathrm{~b}$ : Saint-Pierre-aux-.Vonnains, une nouvelle datation par l'archéomagnétisme, Les Cahiers Lorrains, 2, p. 195-198.

\section{Desbat A.}

1984 : Les Fouilles de la rue des Farges à Lyon, 1974-1980, Lyon. Éd. Groupe Lyonnais de Recherche en Archéologie Galloromaine, 107 p., 121 fig.

Drack W.

1986: Römische Wandmalerei aus der Schweiz, Feldmeilen. Raggi-Verlag. 87 p., 66 fig.. 16 pl. coul.

Duncan-Jones R. P.

1980 : Length-Units in Roman Town Planning: The Pes Monetalis and the Pes Drusianus. Britannia, XI. p. 127-1333.
Fremersdorf F.

1956: Das römische Haus mit dem Itonysos-.Mosaik vor dem südportal des Kölner Domes, Berlin, Verlag Gebr. Mann.

Frere S. S.

1977 : Town Planning in the Western Provinces, Festschrift zum 75. jührigen Beslehen der Römisch-Germanischen Kommission. Beiheft zum Bericht der Römisch-Germanischen Kommission, 58, p. 87-10:3.

Frézouls E., dir.

1982 : Metz antique, in: Les villes antiques de la France. 1. Belgique 1. Strasbourg. Éd. Association pour l'étude de la civilisation romaine. p. 2:35-3500.

Goudineau Chr.

1980: Les villes de la paix romaine. in: (i. Duby (dir.). IIsistoire de la France urbaine, 1. La ville antique, Paris. seruil, p. 2:333-391.

Goudineau Chr., Kruta V.

1980 : Les antecedents : $y$ a-t -il une ville protohistorique? in : (i. I)uby (dir.). Ilistoire de la France urbaine, 1, La ville antique. Paris, Seuil, p. 1:39-231.

Hatt J.-J.

1960): Informations archéologiques, Circonscription de Strasbourg, Gallia, XVIII, p. 213-220.

1961 : Les fouilles de la basilique Saint-Pierre-aux-. Nonnains de Met\% en 1966. Annuaire de la Socièlé d'Ilistoire et d'Archéologie de la Lorraine. L.XI. p. 15-26.

1962: Informations archéologiques, Circonscription de Strasbourg, (iallia, XX, p. 492-496.

1964 : Relevé des niveaux antiques sur le chantier du parking souterrain. Annuaire de la Société d'Ilistoire el d'.Archéologie de la Lorraine. I.XIV, p. 77-85.

Heckenbenner D.

198:3 : Peintures murales en Lorraine: essai de classification. in : La peinlure murale romaine dans les provinces de l'Empire. BAR International series, 165, p. 167-176.

\section{Jolin R.}

1977: Vestiges romains entre la rue des Cleres et Vexirue a Met\%. Innuaire de la socielé d'llistoire et d'Archéologie de la Lorraine, LXXXVII, p. 17-26.

Keune J. B.

19(0): Bericht über die Erwerbungen des Museums der Stadt Metz. Jahrbuch der Gesellschaft für lolhringische Geschichte und Alterlumskinde, 12, p. 346-416.

1904: Aus einem Bericht über Altertumsfunde in Metz und Lothringen. Jahrbuch der (jesellschaft für lolhringische Geschichle und dlerlumskunde. 16. 1. 477-18:3.

\section{Lauranceau N., Maurin L.}

1988 : Structures, caractères, étapes de l'occupation, des origines au Bas-Empire, in : Les fouilles de "Ma maison", Eludes sur Saintes Antique, 3" suppl. à Aquitania, p. 7-71. 
Lefebvre C., Wagner P.

198.1 : Metz antique. Remarques sur la connaissance de l'organisation spatiale du fait urbain. in: Les Villes de la Gaule Belgique au IIaut-Empire. Artes du Colloque lenu à Saint-Riquier (Somme) les 22-24 octobre 1982. Revue Archéologique de Picardie. $3-4$, p. 119-169.

\section{Leroy M. et alii}

1990 : Contribution à l'êtude de la sidérurgie antique : un atelier en milieu urbain à Met $\%$ (Arsenal Ney). Revue Archéologique de l'Est et du Centre-Est. 41, p. 289-300.

\section{Mangin M.}

1981 : I'n quartier de commerģants et d'artisans d'Alésia. Contribution à l'histoire de l'habilat urbain en Gaule. Bibliothèque Pro Alesia VIII. Éd. Lniv. de Dijon.

\section{Morel J.}

1988: Vouvelles données sur l'urbanisme d'Aventicum. les fouilles "St Martin" et ".Mur des Sarrazins" de 1986. Bulletin de P.Association I'ro Aventico $\mathrm{n}^{\circ} 30$.

\section{Peacock D. P. S., Williams D. F.}

1986: Amphorae and the Roman Economy, an introduction guide, Londres. Longman Arrhaeology Series.

Pepe C.

1990: Quand la spectrométrie de masse détermine la fonction de caniveaux gallo-romains.... in : Les mystères de l'archéologie. Les Sciences a la Recherche du Passé. catalogue d'exposition, Presses Iniversitaires de Lyon, Caisse Nationale des Monuments Historiques et des Sites. p. 228.

Petit J.-P., Schaub J.

1990 : Le vicus de Bliesbruck, in : La Lorraine antique, villes el villages. ouvrage édite a l'occasion de l'exposition au Musée d'Art ef d'Histoire de Met\% p. 130-134.

Precht G.

1971: Die Ausgrabungen um den Kölner Dom. Vorbericht über die Intersuchungen 1969/70. Kölner Jahrbuch für Vorund Frühgesshichte. 12. p. 52-64.

\section{Reusch W.}

1943a : Die s. Peter Basilika auf der Zitadelle in .let 7. Germania, XXVII, p. 79-9\%.

1943b : Met\% als Herstellungsort belgischer Keramik. Die Töpferei des Casicos, Germania. XXVII. p. 146-156.

1944 : Der Töpfer CAsICOs, Bodenständiges Töpferhandwerk in Metz vor zweitausend Jahren, Erbe und Heimat, I. p. 39-12. 1955: Die Aula Palatina in Trier, Germania, 33, p. 180-199.

\section{Rouen gallo-romain}

1982: Rouen gallo-romain. Fouilles et recherches archéologiques. 1978-1982. catalogue d'exposition. Musée des Beaux-Arts de Rouen, $114 \mathrm{p}$.

Sabrié M., Sabrié R., Solier Y.

1987 : La Maison à Porliques du Clos de La Lombarde à Varbonne el sa décoration murale. Paris, Ed. C.NRS, 16r suppl. a la Renue Archéologique de Varbonnaise, 372 p., 257 fig.. 3 dépliants. 9 pl. coul.

\section{Stachowski P.}

1990: Hettange-Grande, in : La Lorraine antique, villes et villages, ouvrage édité a l'occasion de l'exposition au Musée d'Art et d'Histoire de Metz. p. 154-158.

\section{Wacher $\mathbf{J}$.}

1974: The Towns of Roman Britain. Londres, B.T. Batsford L.'. 160 p.. 89 fig.

Walthew C. V.

1978 : Property - Boundaries and the Sizes of Building - Plots in Roman Towns, Brilannia, IX, p. 335-350.

\section{Waton M.-D.}

1986 : Metz: Pontiffrov (Moselle). Sauvetage 1983-1985. Revue Archeologique de l'Est el du Centre-Est. XXXVII. 1-2, p. 75-97. 